\title{
A check-list and zoogeographic analysis of the spider fauna (Arachnida: Aranei) of Novosibirsk Area (West Siberia, Russia)
}

\author{
Список и зоогеографический анализ фауны пауков (Arachnida: \\ Aranei) Новосибирской обцасти (Западная Сибирь, Россия)
}

\author{
G.N. Azarkina ${ }^{1}$, I.I. Lyubechanskiii ${ }^{1}$ L.A. Trilikauskas ${ }^{1}$, R.Yu. Dudko', \\ A.N. Bespalov', V.G. Mordkovich ${ }^{1}$ \\ Г.Н. Азаркина ${ }^{1}$, И.И. Аюбечанский ${ }^{1}$, А.А. Трицикаускас ${ }^{1}$, \\ Р.Ю. Аудко ${ }^{1}$, А.Н. Беспалов ${ }^{2}$, В.Г. Мордкович ${ }^{1}$
}

\begin{abstract}
${ }^{1}$ Institute of Systematics and Ecology of Animals SB RAS (ISEA), Frunze str. 11, Novosibirsk 630091, Russia. E-mail: urmakuz@gmail.com Институт систематики и экологии животных СО РАН, ул. Фрунзе, 11, Новосибирск 630091, Россия.

${ }^{2}$ Institute of Soil Science and Agrochemistry SB RAS, Lavrentiev Avenue 8/2, Novosibirsk 630090, Russia.

Институт почвоведения и агрохимии СО РАН, проспект Лаврентьева 8/2, Новосибирск, 630090, Россия.
\end{abstract}

KEY WORDS: Araneae, diversity, natural complexes, ranges, spiders, Carabidae.

КЛЮЧЕВЫЕ СЛОВА: Araneae, ареалы, пауки, природные комплексы, разнообразие, жужелицы.

ABSTRACT. A check-list of the spiders (Arachnida, Aranei) recorded from Novosibirsk Area (364 species in 157 genera and 26 families) is provided, with the references to exact collection localities, administrative units, natual complexes, and latitudinal \& longitudinal components of their ranges. Of the reported spiders, 164 species, 53 genera and three families, including two new species that are being currently described, have been recorded from the Area for the first time. A large number of the species having the northernmost or morth-easternmost limits of their distribution in Novosibirsk Area is indicative of the importance of the latitudinal boundary occurring on its territory and also of the similarity of spider faunas between the south part of Novosibirsk Area and Kazakhstan. It is also possible that the penetration of southern species northward during the latest decades is related to the warming and aridization of climate in West Siberia, as shown for other invertebrate groups. Based on the ShimkevichSimpson Similarity Index, the spider faunas of six provinsional units (natural complexes) of Novosibirsk Area are grouped in two clusters: those of left and right banks of Ob' River. The faunas of the left bank forest and forest-steppe (Baraba) subzones are close and distinct from those of the right bank faunas. In the right bank cluster, an anticipated similarity of Priobsky faunas is observed, with the araneofauna of the Salair Ridge standing apart. The subdivision and zoogeographic regioning of local ground-beetle faunas of Novosibirsk Area is approximately the same as those revealed for spiders.

How to cite this article: Azarkina G.N., Lyubechanskii I.I., Trilikauskas L.A., Dudko R.Yu., Bespalov A.N., Mordkovich V.G. 2018. A check-list and zoo- geographic analysis of the spider fauna (Arachnida: Aranei) of Novosibirsk Area (West Siberia, Russia) // Arthropoda Selecta. Vol.27. No.1. P.73-93. doi: 10.15298/arthsel. 27.1.11

РЕЗЮМЕ. Дан список пауков, зарегистрированных в Новосибирской области (364 вида из 157 родов и 26 семейств), с указанием локалитетов, привязкой к границам административных районов и природных комплексов и указанием широтной и долготной компоненты ареала. На территории области мы впервые отмечаем 164 вида, 53 рода и три семейства пауков, в том числе два вида - новые для науки и находятся сейчас в процессе описания. Большое число видов пауков, имеющих в Новосибирской области северную или северо-восточную границу распространения, показывает значимость широтного рубежа, проходящего по ее территории, и говорит о высокой общности фаун пауков юга Новосибирской области и Казахстана. Возможно также продвижение южных видов на север, связанное с потеплением и аридизацией климата в Западной Сибири в последние десятилетия, как это наблюдается в других группах беспозвоночных. Фауны пауков шести провинциальных выделов (природных комплексов) Новосибирской области по своему сходству (коэффициент Шимкевича-Симпсона) разделились на две части - левобережную и правобережную. Фауны левобережных лесной и Барабинской лесостепной областей достаточно близки и хорошо отделяются от фаун правобережной части. В ней наблюдается ожидаемое тесное сходство Приобских фаун, особняком от них стоит фауна Салаирских предгорий. Примерно так же, 
как у пауков, выглядит и зоогеографическое районирование, проведенное по локальным фаунам жуков-жужелиц для Новосибирской области.

\section{Introduction}

Novosibirsk Area (Oblast'), zoogeographically, is a model region in whole West Siberia. Its central geographic position, plain relief and a combination of virtually flat, closed (undrained) regions with large rivers facilitate deep interpenetration and mixing up of western and eastern, northern and southern faunas on this territory. The study of the araneofauna of Novosibirsk Area helps us to better understand the patterns of zoogeographic and landscape distribution of regional biota, and the faunistic (inter)connections between spiders of the Urals, western and eastern Siberia, the Altai and Central Asia.

Novosibirsk Area is situated in the south-eastern part of West Siberian Plain, the largest one in Eurasia, with the area of about 178,200 square $\mathrm{km}$, being almost equal to the territories of such countries as Cambodia $\left(181,040 \mathrm{~km}^{2}\right)$ or Syria $\left(185,180 \mathrm{~km}^{2}\right)$. The territory of Novosibirsk Area is extended for over $600 \mathrm{~km}$ from the west to the east, and for over $400 \mathrm{~km}$ from the north to the south. Its relief is predominantly flat, with absolute heights ranging from 100 to $200 \mathrm{~m}$ a.s.l., except for the Salair Ridge with heights up to $500 \mathrm{~m}$ a.s.1., which is situated in the south-eastern part of Novosibirsk Area and represents the north-western outpost of the mountains of South Siberia. The territory of Novosibirsk Area includes the forest (southern taiga and sub-taiga), forest-steppe and steppe natural zones. Yet, there are vast territories of intrazonal landscapes, such as extensive Vasyugan raised moors in the north, the wide valley of Ob' River with a complex mosaic of inundated forests, meadows and bogs, the salt marshes near the undrained Lake Chany (largest in West Siberia). Large rivers run not only in the meridional direction, as Ob' River, but also in the zonal direction, as rivers Tara, Tartas, Om', Kargat, Chulym, Karasuk, Inya and Berd'.

Although the spider fauna of Novosibirsk Area is studied less thoroughly than those of Altai Territory, the Republic of Altai or Tuva [cf. Azarkina, Trilikauskas, 2012, 2013a,b, Marusik et al., 2000], its current state of knowledge is good enough in order to assess its diversity and to undertake a zoogeographic analysis for the first time. The relatively good state of knowledge of the spider fauna at hand could be explained by the presence of a large academic centre in the region and a long standing entomological collecting in its vicinity (City of Novosibirsk and Novosibirsk District). Longterm studies of spider fauna were also carried out in the ISEA field stations 'Karasuksky' (Karasuk Distr., steppe zone; 2007-2008 and following years) and 'Chanovsky' (Zdvinsk Distr., central forest-steppe; 2009-2010).

The aims of the present study are (1) to inventory the spider fauna of Novosibirsk Area, and (2) to carry out its zoogeographic analysis.

\section{Study area, material and methods}

The present study is based on the spider collections deposited in the Institute of Systematics and Ecology of Animals SB RAS (ISEA). These collections have been assembled by many researchers from 113 localities of Novosibirsk Area in 1976-2017 (see Map).

Besides examining museum collections, the authors also carried out own fieldworks. Spiders were primarily collected by pitfall traps and also sorted out from soil samples from the vicinities of 'Karasuksky' and 'Chanovsky' field stations: the localities Troitskoe (Map: 106) and Shirokaya Kur'ya (Map: 86) correspondingly. In many other localities the authors handcollected spiders as well.

Novosibirsk Area consists of the City of Novosibirsk and 30 administrative units, of which borders are shown on the Map. Each mapped locality is named by its closest settlement and numbered continuously.

We have used the physiogeographic subdivision of Novosibirsk Area in four provinces, which are further subdivided in nine landscape subzones (=natural complexes) [Atlas..., 2002: map 'Natural complexes' on $p$. 31]. The Forest Province is subdivided in Vasyugan taiga-swampy (Map: I), Priobsky south taiga (II), North Baraba sub-taiga (III), and Priobsky birch-aspen forest (IV) subzones. The Forest-Steppe Province is subdivided in Baraba (V) and Priobsky (VI) subzones. The Steppe Province (VII) is not subdivided. The Kuznetsk-Salair Province is subdivided in Salair forest (VIII) and Kuznetsk hollow forest-steppe (IX) subzones. Borders of these landscape zones are shown on the Map.

Species collected from the localities situated near borders of the landscape subzones are listed in both

Новосибирск: 56 - Новосибирск; IS - Искитимский район: 57 - Морозово, 58 - Александровка, 59 - Искитим, 60 Шипуново, 61 - Харино, 62 - Белово, 63 - Легостаево, 64 - Малиновка, 65 - Новососедово, 66 - Завьялово, 67 Быстровка, 68 - Евсино, 69 - Койниха; ТО - Тогучинский район: 70 - Гутовский, 71 - Тогучин, 72 - Буготак, 73 Горный, 74 - Буготакские сопки, 75 - Карпысак, 76 - Вассино, 77 - Лебедево, 78 - Мирный, 79 - Которово, 80 - оз. Танаево; СІ - Чистоозёрный район: 81 - оз. Большое Горькое, 82 - оз. Кулмакан, 83 - оз. Солёное (Новокрасное), 84 Золотая грива (Новокрасное); KU - Купинский район: 85 - Сибирский; ZD - Здвинский район: 86 - Широкая Курья; DO — Доволенский район: 87 - Индерь; КK - Кочковский район: 88 - Кочки, 89 - Черновка; ОR - Ордынский район: 90 Верх-Ирмень, 91 - Новый Шарап, 92 - Ордынское, 93 - Нижнекаменка, 94 - Верх-Алеус, 95 - Усть-Алеус; SU — Сузунский район: 96 - Сузун; СЕ - Черепановский район: 97 - Дорогино, 98 - Черепаново, 99 - Бочкарёво; МА Маслянинский район: 100 - Верх-Ики, 101 - Берёзово, 102 - Кинтереп, 103 - Никоново, 104 - Маслянино; ВG Баганский район: 105 - Новоключи; КА - Карасукский район: 106 - Троицкое, 107 - Карасук, 108 - Астродым, 109 Студёное, 110 - оз. Чаган; KR - Краснозёрский район: 111 - Кайгородский, 112 - Краснозёрское, 113 - Половинное. 


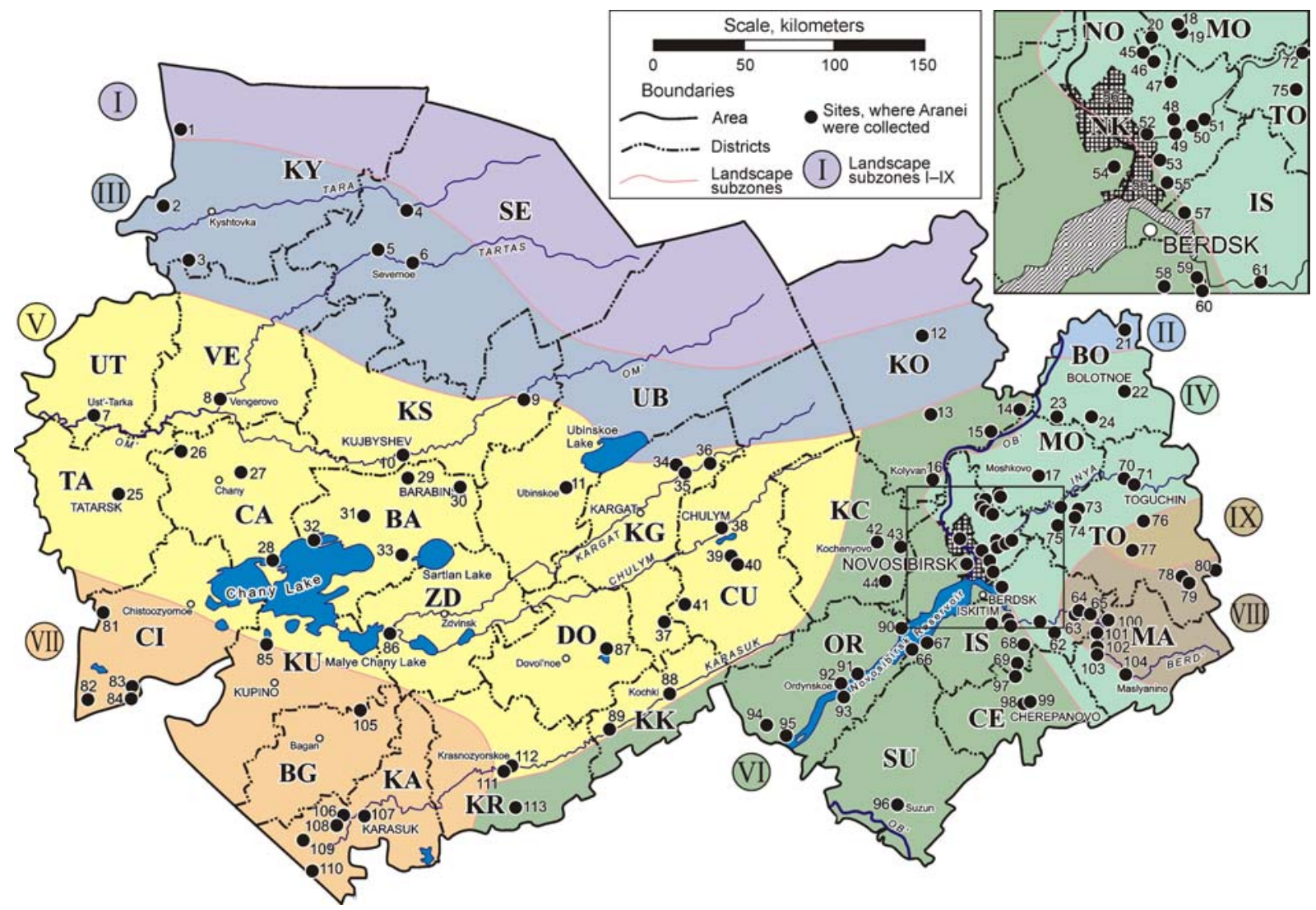

Map. Collecting localities of spiders across administrative districts (rajons) of Novosibirsk Area.

KY - Kyshtovka District: 1 - Orlovka, 2 - Bol'sherech'e, 3 - Usmanka; SE - Severnoe District: 4 - Biaza, 5 - Malinovka, 6 - Severnoe; UT - Ust'-Tarka District: 7 - Ust'-Tarka; VE - Vengerovo District: 8 - Vengerovo; KS - Kujbyshev District: 9 - Zonovo, 10 - Kujbyshev; UB - Ubinskoe District: 11 - Ubinskoe; KO - Kolyvan' District: 12 - Pikhtovka, 13 - Boyarka, 14 - Umna, 15 - Chyornyi Mys, 16 - Kolyvan'; MO - Moshkovo District: 17 - Moshkovo; 18 - Sokur, 19 - Smolenka, 20 Oktyabr'skii; BO - Bolotnoe District: 21 - Bol'shaya Chyornaya, 22 - Bolotnoe, 23 - Oyash, 24 - Chebula; TA - Tatarsk District: 25 - Tatarsk; CA - Chany District: 26 - Uzungul', 27 - Karachi, 28 - Chany Lake; BA - Barabinsk District: 29 Barabinsk, 30 - Penzino, 31 - Tandovo Lake, 32 - Chany Lake, 33 - Kozhevnikovo; KG - Kargat District: 34 - Rovenskoe, 35 - Verkh-Kargat, 36 - Makar'evskii, 37 - Alabuga; CU - Chulym District: 38 - Chulym, 39 - Sherstobitovo, 40 - Filimonovo, 41 - Sarykamyshka; KC - Kochenyovo District: 42 - Kochenyovo, 43 - Chik, 44 - Antonovka; NO - Novosibirsk District: 45 Mochishche, 46 - Leninskii, 47 - Zherebtsovo, 48 - Uchebnyi, 49 - Izdrevaya, 50 - Sovkhoznaya, 51 - Shelkovichikha, 52 Novolugovskoe, 53 - Kol'tsovo, 54 - Krasnoobsk, 55 - Shadrikha; NK - Novosibirsk city: 56 - Novosibirsk; IS - Iskitim District: 57 - Morozovo, 58 - Aleksandrovka, 59 - Iskitim, 60 - Shipunovo, 61 - Kharino, 62 - Belovo, 63 - Legostaevo, 64 Malinovka, 65 - Novososedovo, 66 - Zav'yalovo, 67 - Bystrovka, 68 - Evsino, 69 - Koynikha; TO - Toguchin District: 70 Gutovskii, 71 - Toguchin, 72 - Bugotak, 73 - Gornyi, 74 - Bugotakskie sopki, 75 - Karpysak, 76 - Vassino, 77 - Lebedevo, 78 - Mirnyi, 79 - Kotorovo, 80 - Tanaevo Lake; CI - Chistoozyornoe District: 81 - Bol'shoe Gor'koe Lake, 82 - Kulmakan Lake, 83 - Solyonoe Lake (Novokrasnoe), 84 - Zolotaya griva (Novokrasnoe); KU - Kupino District: 85 - Sibirskii; ZD - Zdvinsk District: 86 - Shirokaya Kur'ya; DO — Dovol'noe District: 87 - Inder'; KK — Kochki District: 88 - Kochki, 89 - Chernovka; OR — Ordynskoe District: 90 - Verkh-Irmen', 91 - Novyi Sharap, 92 - Ordynskoe, 93 - Nizhnekamenka, 94 - Verkh-Aleus, 95 Ust'-Aleus; SU - Suzun District: 96 - Suzun; CE - Cherepanovo District: 97 - Dorogino, 98 - Cherepanovo, 99 - Bochkaryovo; MA - Maslyanino District: 100 - Verkh-Iki, 101 - Beryozovo, 102 - Kinterep, 103 - Nikonovo, 104 - Maslyanino; BG - Bagan District: 105 - Novoklyuchi; KA — Karasuk District: 106 - Troitskoe, 107 - Karasuk, 108 - Astrodym, 109 - Studyonoe, 110 Chagan Lake; KR — Krasnozyorskoe District: 111 — Kajgorodskii, 112 — Krasnozyorskoe, 113 — Polovinnoe.

Карта. Точки сборов пауков по административным районам Новосибирской области.

$\mathbf{K Y}$ - Кыштовский район: 1 - Орловка, 2 - Большеречье, 3 - Усманка; $\mathbf{S E}-$ Северный район: 4 - Биаза, 5 Малиновка, 6 - Северное; UT - Усть-Тарский раойн: 7 - Усть-Тарка; VE - Венгеровский район: 8 - Венгерово; KS Куйбышевский район: 9 - Зоново, 10 - Куйбышев; UB - Убинский район: 11 - Убинское; КО - Колыванский район: 12 - Пихтовка, 13 - Боярка, 14 - Умна, 15 - Чёрный мыс, 16 - Колывань; МО - Мошковский район: 17 - Мошково; 18 Сокур, 19 - Смоленка, 20 - Октябрьский; ВО - Болотнинский район: 21 - Большая Чёрная, 22 - Болотное, 23 - Ояш, 24 - Чебула; ТА - Татарский район: 25 - Татарск; СА - Чановский район: 26 - Узунгуль, 27 - Карачи, 28 - оз. Чаны; $\mathbf{B A}$ - Барабинский район: 29 - Барабинск, 30 - Пензино, 31 - оз. Тандово, 32 - оз. Чаны, 33 - Кожевниково; KG Каргатский район: 34 - Ровенское, 35 - Верх-Каргат, 36 - Макарьевский, 37 - Алабуга; $\mathbf{C U}$ - Чулымский район: 38 Чулым, 39 - Шерстобитово, 40 - Филимоново, 41 - Сарыкамышка; КС - Коченёвский район: 42 - Коченёво, 43 - Чик, 44 - Антоновка; NO - Новосибирский район: 45 - Мочище, 46 - Ленинский, 47 - Жеребцово, 48 - Учебный, 49 - Издревая, 50 - Совхозная, 51 - Шелковичиха, 52 - Новолуговское, 53 - Кольцово, 54 - Краснообск, 55 - Шадриха; NK - 


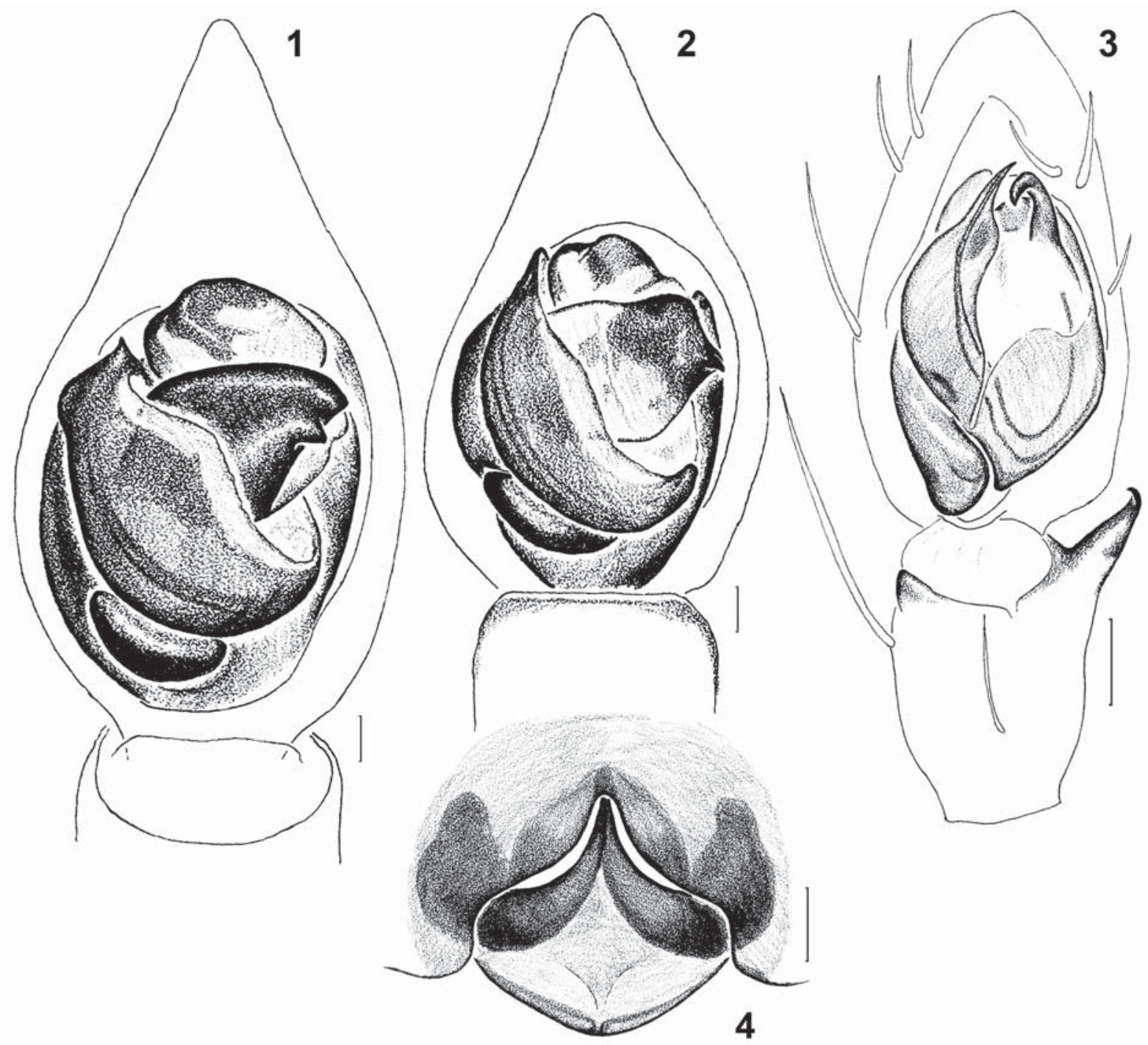

Figs 1-4. Copulatory organs, ventral view: $1-$ Alopecosa taeniopus (Kulczyński, 1895), palp; $2-$ A. kovblyuki Nadolny et Ponomarev, 2012, palp; 3 - Gnaphosa ukrainica Ovtsharenko, Platnick et Song, 1992, palp; 4 - A. taeniopus, epigyne. Scale bars $=0.1 \mathrm{~mm}$.

Рис. 1-4. Копулятивные органы, вид снизу: 1 - Alopecosa taeniopus (Kulczyński, 1895), пальпа; 2 - A. kovblyuki Nadolny et Ponomarev, 2012, пальпа; 3 - Gnaphosa ukrainica Ovtsharenko, Platnick et Song, 1992, пальпа; 4 - A. taеnіориs, эпигина. Масштаб 0,1 мм.

neighbouring faunas. For instance, the fauna of Novosibirsk (site 56) is included in those of both Priobsky forest-steppe (VI) and Priobsky birch-aspen forest (IV) subzones.

A range analysis of the araneofauna of Novosibirsk Area has also been carried out; it is based on the range typology elaborated for the ground beetles (Coleoptera, Carabidae) [Dudko, Lyubechanskii, 2002; Bespalov et $a l ., 2010]$ that has been adopted for spiders [Mordkovich et al., 2015; Trilikauskas, Dudko, 2016; Lyubechanskii, Azarkina, 2017]. Latitudinal and longitudinal components of each species range are considered separately.

With regard to latitudinal components, the following four groups have been considered: boreal, subboreal humid, subarid and polyzonal. The northern limit of the boreal group lies in the zone of tundra/forest-tun$\mathrm{dra}$; in the south, boreal species can reach the steppe zone in the central sector of Palaearctic Region or to the zone of broad-leaved forests in its western and eastern sectors. Boreomontane species, which can occur southerly in the mountains, are also considered in the same group. The subboreal-humid group includes species that are not found north of the subzone of the middle taiga in central sector of Palaearctic Region; the southern limit of these species lies in the steppe zone. The subarid group includes species that are limited in the north by the forest-steppe zone. The distribution of polyzonal species in the north is similar to that of boreal species; in the south, they can reach the zone of semi-deserts or even southerly. 
With respect to longitudinal components, we have grouped species ranges based on their relation to the boundaries of pan-Atlantic, continental or pan-Pacific sectors of the Palaearctic Region [sensu Emelyanov, 1974]. Spider species have been considered in five latitudinal groups: trans-Holarctic (i.e., occurring in all sectors of the Palaearctics and Nearctics), trans-Palaeartic (i.e., occurring in all three sectors of the Palaearctics), west-Palaearctic (i.e., occurring in pan-Atlantic and continental sectors of the Palaearctics), centralPalaearctic (i.e., occurring in the continental sector only), and east-Palaearctic (i.e., occurring in the continental, pan-Pacific and Nearctic sectors).

Some spider species with poorly known distribution have been formally considered endemics: viz., Asianellus kazakhstanicus Logunov et Heçiak, 1996, Evippa sp. and Xysticus sp. (see Table 1). Two synantropic species, Pholcus opilionoides (Schrank, 1871) and Steatoda grossa (C.L. Koch, 1838), are given in the Table as polyzonal west- and trans-Holarctic ones coresponsingly. The natural range of $P$. opilionoides seems to be restricted by the Caucasus [Huber, 2011], whereas $S$. grossa seems to have a south-Palaearctic origin, being currently widespread in North and South Americas [Levi, 1967; World Spider Catalog, 2017].

The spider taxonomy follows World Spider Cata$\log$ [2017], except for Allohogna singoriensis (Laxmann, 1770) and Styloctetor stativus Simon, 1881; distribution patterns are primarily based on Mikhailov [2013] and World Spider Catalog [2017].

Since in the boundaries of provinces and natural complexes occurring in the territory Novosibirsk Area are mostly latitudinal, in the following zoogeographic analysis a more attention is paid to the latitudinal components of spider ranges. An analysis of the longitudinal components will be of interest in a larger-scale study while comparing Novosibirsk Area with neighbouring territories.

A similarity of the studied faunas has been assessed by the Szymkiewicz-Simpson index (ISzS) and then ordinated by the multidimensional scaling (MDS) in the programme PAST 2.17 [Hammer et al., 2001].

\section{Results and discussion}

History of arachnological studies in Novosibirsk Area

The investigations of spiders of the territory that would later be included in present-day Novosibirsk Area (founded on 1937) started from the work by Chugunov \& Chugunov [1902] in which the authors reported on Allohogna singoriensis (Laxmann, 1770) (sub: Lycosa latreili) from Tatarsk. Two following works published 20 years later also dealt with records of the same species from Karachi Lake [Lavrova, 1923: sub Trochosa s.; Ruzski, 1924: sub. Trochosa syngoriensis]. A year later, Ruzski [1925] already mentioned two spider species from the vicinity of Lake Karachi: i.e.,
A. singoriensis (sub: Trochosa syngoriensis) and Argyroneta aquatica (Clerck, 1758), from the families Lycosidae and Cybaeidae correspondingly. Three years later, two papers devoted to spiders of Lake Karachi were published: Ruzski [1928] who mentioned A. singoriensis once more, and Ermolaev [1928] who reported on 14 species of 12 genera in eight families. One species of the latter work, Alopecosa mariae (F. Dahl, 1908), is excluded from the current species list of Novosibirsk Area, as the original identification was based on a single female and is likely to actually belong to Alopecosa taeniopus (Kulczyński, 1895), a common spider species in forest-steppe habitats. The remaining taxa recorded by Ermolaev [1928], except for A. singoriensis (sub: Hogna singoriensis), were new to the Region - 12 species in 11 genera and seven families. In two followings papers [Ermolaev, 1930; Ruzski, 1946], only a few additional records $A$. singoriensis were provided.

The next arachnological paper devoted to spiders of Novosibirsk Area was published 31 years later [Lobanova, 1976], dealing with new records of wolf-spiders. The latter paper provides data on 31 species in seven genera, of which several species are to be excluded from the current species list. Part of the records of Pardosa calida Blackwall, 1875 (from Moshkovo) are to be referred to P. bifasciata C.L. Koch, 1836.) nowadays, the name $P$. calida is not valid [WSC, 2017]. Pardosa monticola (Clerck, 1758) seemed to be identified incorrectly and its records are to be referred to $P$. agrestis (Westring, 1861), P. palustris (Linnaeus, 1758) or $P$. plumipes (Thorell, 1875); yet, this species is temporarily retained as a valid record for Novosibirsk Area (Table 1). The record of Alopecosa solitaria (Herman, 1879) from Barabinsk seems to be that of either A. sulzeri (Pavesi, 1873) or A. taeniopus [Esyunin, pers. comm.]. The identification of Alopecosa accentuata (Latreille, 1817) (in Lobanova [1976] and Lyubechanskii \& Azarkina [2017]) is to be referred to $A$. farinosa (Herman, 1879). Thus, Lobanova [1976] recorded 28 species in five genera of wolf-spiders from Novosibirsk Area for the first time. In the following series of papers [Lobanova, 1980, Lobanova, Ryabikova, 1980, Ryabikova, Lobanova, 1980], 29 spider species of 14 genera in three families were mentioned, of which 15 species in 14 genera and three families (Araneidae, Linyphiidae and Lycosidae) were recorded from the Area for the first time. In two papers by Ryabikova [1985, 1989] devoted to the Araneidae, ten species in six genera were first reported for the Area. The locality Maletino given for Araneus diadematus (Clerck, 1758) (sub: Malitino in Ryabikova [1985]) is to be excluded, for it is situated in Altai Territory. The locality Larino [Ryabikova, Lobanova, 1980] was misspelled and is to be read as Kharino [Ryabikova, pers. comm.]. Thus, for the almost 90-year period (1902-1989), 67 spider species (excluding Alopecosa mariae and A. solitaria) in 32 genera and ten families have been recorded from Novosibirsk Area. 
From 1990 to 1999 the number of spider species found in Novosibirsk Area had been significantly increased. Representatives of the families Clubionidae (one genus and four species; Mikhailov [1992]) and Salticidae (26 species in 16 genera [Logunov, 1992a, 1999; Danilov, Logunov, 1994; Logunov, Heçiak, 1996; Logunov, Kronestedt, 1997; Logunov, Marusik, 1994, 1999; Logunov, Rakov, 1996; Logunov, Wesołowska, 1995; Logunov et al., 1993; Marusik, Logunov, 1998; Mikhailov, 1996; Rakov, 1999]) were recorded for the first time. In three families the number of recorded species was also increased: Gnaphosidae - by one genus and species [Ovtsharenko et al., 1992], Philodromidae - by one genus and six species [Logunov, 1996], and Thomisidae - by one genus and two species [Logunov, 1992b; Logunov, Marusik, 1998]. Eskov \& Marusik [1992, 1994] reported on 15 species in 14 genera of the family Linyphiidae, of which 14 species in 13 genera were new to the Area. Of the orbweavers (Araneidae), Larinia jeskovi Marusik, 1986 was first found in the area at hand [Logunov, 1990]. Thus, during less than a ten-year period the number of recorded species and genera had been almost doubled and reached 120 species in 66 genera. The number of recorded families had been increased from ten to 12 .

In the latest period (2000-2017), 86 additional spider species in 44 genera and 11 families have been recorded from the Area by various authors [Azarkina, Logunov, 2001; Azarkina et al., 2015; Fyodorov, Trilikauskas, 2013; Logunov, Marusik, 2000a,b; Marusik et al., 2007; Marusik, Omelko, 2014; Mikhailov, 2003; Szita, Logunov, 2008; Tanasevitch, 2000; Trilikauskas, Dudko, 2016; Lyubechanskii, Azarkina, 2017; Lyubechanskii et al., 2017]. The record of Heliophanus dubius C.L. Koch, 1835 by Litvinchuk [1980] for Kulunda steppe was made without referring to an exact locality; it was listed as that from Novosibirsk Area by Logunov \& Marusik [2000b], but actually belongs to Altai Territory and is thus excluded from the present account. Four literature salticid records - Evarcha arcuata (Clerck, 1758), Heliophanus flavipes (Hahn, 1832), Marpissa radiata (Grube, 1859) and Sitticus floricola (C.L. Koch, 1837) — were mentioned by Logunov \& Marusik [2000b] as being made from the vicinity of Karachi, but in the original paper [Ermolaev, 1928] they were actually referred to Kuznetsk Hollow which lies outside present day Novosibirsk Area. Two species identified as Alopecosa osa Marusik, Hippa et Koponen, 1996 and Gnaphosa species [Fyodorov, Trilikauskas, 2013; Mordkovich et al., 2015] are to be referred to Alopecosa kovblyuki Nadolny et Ponomarev, 2012 (see Fig. 2) and Gnaphosa ukrainica Ovtsharenko, Platnick et Song, 1992 (see Fig. 3). The record of Xysticus idolothytus Logunov, 1995 [Fyodorov, Trilikauskas, 2013; Mordkovich et al., 2015] belongs to a new species that is indicated in Table 1 as Xysticus sp. Thus the names Alopecosa osa and Xysticus idolothytus have been excluded from the current check-list. The record of Alopecosa solitaria by Ly- ubechaskii \& Azarkina [2017] is actually that of $A$. taeniopus (see Figs 1, 4).

In the present work, 164 species in 53 genera and three families have been recorded from Novosibirsk Area for the first time. By this means, a total of 364 spider species in 157 genera and 26 families has been registered from the studied area to date. Of them, two species are new to science and their descriptions are currently under preparation. A complete check-list of the registered species with all their records according to districts (see Map) and with comments on latitudinal and longitudinal components of their ranges are given in Table 1.

Clarification of spider ranges within the scope of West Siberia

In the present work, 48 species in 33 genera and five families have been recorded from West Siberia (the physiographic region 'M' sensu Mikhailov [2013]; i.e., except for the mountains of South Siberia) for the first time. On the territory of Novosibirsk Area the records of nine species represent the northernmost limits of their ranges: Alopecosa azsheganovae Esyunin, 1996, Argyope lobata (Pallas, 1772), Chalcoscirtus nigritus (Thorell, 1875), Heriaeus horridus Tyschchenko, 1965, Oxyopes xinjiangensis $\mathrm{Hu}$ et $\mathrm{Wu}, 1989$, Pellenes epularis (O. Pickard-Cambridge, 1872), Sauron fissocornis Eskov in Eskov et Marusik, 1995, Xysticus pseudocristatus Azarkina et Logunov, 2001 and Walckenaerianus esyunini Tanasevitch, 2004. The northeasternmost records have been made for the following seven species: Alopecosa kasakhstanica Savelyeva, 1972, A. kovblyuki Nadolny et Ponomarev, 2012, Chalcoscirtus brevicymbialis Wunderlich, 1980, Gnaphosa jucunda Thorell, 1875, G. ukrainica Ovtsharenko, Platnick et Song, 1992, Urozelotes trifidus Tuneva, 2003 and Uralophantes troitskensis Esyunin, 1992. The easternmost records have been made for eight species: Agroeca dentigera Kulczyński, 1913, Archaraeoncus prospiciens (Thorell, 1875), Archaeodictyna ammophila (Menge, 1871), Centromerita bicolor (Blackwall, 1833), Dicymbium tibiale (Blackwall, 1836), Haplodrassus minor (O. Pickard-Cambridge, 1879), Moebelia penicillata (Westring, 1851) and Robertus heydemanni Wiehle, 1965. The north-westernmost localities have been registered for Dictyna ubsunurica Marusik et Koponen, 1998 and Gnaphosa tuvinica Marusik et Logunov, 1992. The westernmost record has been made for Philodromus utotchkini Marusik, 1991.

Thus, the majority of new spider records extend the known ranges of corresponding species north-, east- or north-eastward ( 25 of 28 species in total). Two records extend the ranges in a north-westward direction (from Central Asia to West Siberia), and one westward (from the eastern Palaearctics to West Siberia). Such results are partly due to the poor state of knowledge of spiders in some Siberian regions. Nevertheless, a significant 
Table 1. Check-list of spiders of Novosibirsk Area. Таблица 1. Список видов пауков Новосибирской области.

\begin{tabular}{|c|c|c|c|}
\hline Species & LAT & LON & Records in Novosibirsk Area \\
\hline \multicolumn{4}{|c|}{ AGELENIDAE -2 genera, 2 species } \\
\hline Agelena labyrinthica (Clerck, 1758) & $\mathrm{P}$ & TP & 106 \\
\hline Tegenaria*domestica* (Clerck, 1758) & $\mathrm{P}$ & $\mathrm{TH}$ & 56 \\
\hline \multicolumn{4}{|c|}{ ARANEIDAE -14 genera, 30 species } \\
\hline Aculepeira packardi (Thorell, 1875) & $\mathrm{P}$ & TH & 106 \\
\hline Araneus alsine* (Walckenaer, 1802$)$ & $\mathrm{P}$ & $\mathrm{TP}$ & 56 \\
\hline Araneus angulatus Clerck, 1758 & $P$ & $\mathrm{TP}$ & $\begin{array}{l}8,38,51,56,59,66,62,94,91,93,96,98 \\
104,106\end{array}$ \\
\hline Araneus diadematus Clerck, 1758 & $\mathrm{P}$ & $\mathrm{TH}$ & $\begin{array}{l}2,8,17,19,25,44,51,56,71,70,86,93, \\
96,97,106\end{array}$ \\
\hline Araneus grossus (C.L. Koch, 1844) & $\mathrm{SH}$ & WP & 61 \\
\hline Araneus marmoreus Clerck, 1758 & $\mathrm{P}$ & TH & $\begin{array}{l}3,8,13,19,25,44,52,51,53,56,59,64 \\
67,62,71,76,86,94,91,96,106\end{array}$ \\
\hline Araneus nordmanni ${ }^{*}$ (Thorell, 1870$)$ & $\mathrm{P}$ & TH & 56 \\
\hline Araneus quadratus Clerck, 1758 & $\mathrm{P}$ & $\mathrm{TP}$ & $2,3,19,36,64,68$ \\
\hline Araneus sturmi $*(\mathrm{Hahn}, 1831)$ & $\mathrm{P}$ & TP & 56 \\
\hline Araniella displicata $($ Hentz, 1847) & $\mathrm{P}$ & $\mathrm{TH}$ & 106 \\
\hline Araniella proxima* (Kulczyński, 1885) & $\mathrm{B}$ & $\mathrm{TH}$ & 13 \\
\hline Argiope bruennichi (Scopoli, 1772) & $\mathrm{P}$ & $\mathrm{TP}$ & $86,106,108$ \\
\hline Argiope lobata ${ }^{* \mathrm{M}}$ (Pallas, 1772) & $\mathrm{P}$ & WP & 106 \\
\hline Cercidia prominens (Westring, 1851) & $\mathrm{P}$ & TH & $40,56,86$ \\
\hline Cyclosa conica (Pallas, 1772) & $\mathrm{P}$ & TH & $8,14,56,86,94,93,106$ \\
\hline Cyclosa oculata (Walckenaer, 1802) & $\mathrm{P}$ & TP & 25 \\
\hline $\begin{array}{l}\text { Gibbaranea bituberculata (Walckenaer, } \\
\text { 1802) }\end{array}$ & $\mathrm{P}$ & $\mathrm{TP}$ & 56,59 \\
\hline Hypsosinga albovittata* $($ Westring, 1851) & $\mathrm{P}$ & TP & 86,106 \\
\hline Hypsosinga heri (Hahn, 1831) & $\mathrm{P}$ & WP & 86 \\
\hline Hypsosinga pygmaea (Sundevall, 1831) & $\mathrm{P}$ & $\mathrm{TH}$ & $20,33,56,86,106$ \\
\hline Hypsosynga sanguinea (C.L. Koch, 1844) & $\mathrm{P}$ & $\mathrm{TP}$ & 1,19 \\
\hline Larinia jeskovi Marusik, 1986 & SH & TP & $35,40,108$ \\
\hline Larinioides cornutus (Clerck, 1758) & $\mathrm{P}$ & TH & $25,33,56,86,106$ \\
\hline Larinioides ixobolus (Thorell, 1873) & SA & WP & $19,25,56,59,62,86,106$ \\
\hline Larinioides patagiatus (Clerck, 1758) & $\mathrm{P}$ & $\mathrm{TH}$ & $3,8,20,25,29,51,56,59,66,86,106$ \\
\hline $\begin{array}{l}\text { Larinioides suspicax } *(\mathrm{O} \text {. Pickard- } \\
\text { Cambridge, } 1876)\end{array}$ & $P$ & WP & $56,59,84,86,106$ \\
\hline Neoscona adianta (Walckenaer, 1802) & $\mathrm{P}$ & TP & $86,106,109$ \\
\hline Nuctenea silvicultrix (C.L. Koch, 1835) & SH & $\mathrm{TP}$ & 14,59 \\
\hline Singa hamata (Clerck, 1758) & $\mathrm{P}$ & $\mathrm{TP}$ & $19,20,25,33,53,56,59,86$ \\
\hline Singa nitidula C.L. Koch, 1844 & $\mathrm{P}$ & $\mathrm{TP}$ & $19,20,48,56,59$ \\
\hline \multicolumn{4}{|c|}{ CLUBIONIDAE -1 genus, 12 species } \\
\hline Clubiona bashkirica Mikhailov, 1992 & SH & $\mathrm{CP}$ & 9 \\
\hline Clubiona caerulescens L. Koch, 1867 & SH & $\mathrm{TP}$ & 56,113 \\
\hline $\begin{array}{l}\text { Clubiona diversa O. Pickard-Cambridge, } \\
1862\end{array}$ & B & $\mathrm{TP}$ & 53,79 \\
\hline Clubiona frisia Wunderlich et Schuett, 1995 & SH & WP & 56 \\
\hline Clubiona germanica Thorell, 1871 & SH & $\mathrm{TP}$ & 13 \\
\hline Clubiona interjecta* L. Koch, 1879 & $\mathrm{~B}$ & EP & 56 \\
\hline Clubiona lutescens* Westring, 1851 & $\mathrm{SH}$ & $\mathrm{TH}$ & 106 \\
\hline $\begin{array}{l}\text { Clubiona neglecta* } \mathrm{O} . \text { Pickard-Cambridge, } \\
1862\end{array}$ & SH & $\mathrm{TP}$ & 86 \\
\hline Clubiona pallidula (Clerck, 1758) & SH & TH & 53 \\
\hline
\end{tabular}


Table 1 (continued). Таблица 1 (продолжение).

\begin{tabular}{|c|c|c|c|}
\hline Species & LAT & LON & Records in Novosibirsk Area \\
\hline \multicolumn{4}{|c|}{ CLUBIONIDAE } \\
\hline Clubiona phragmitis* C.L. Koch, 1843 & $\mathrm{P}$ & TP & 56,80 \\
\hline Clubiona stagnatilis* Kulczyński, 1897 & $\mathrm{P}$ & WP & 36 \\
\hline Clubiona subtilis L. Koch, 1867 & SH & WP & 36 \\
\hline \multicolumn{4}{|c|}{ CYBAEIDAE -1 genus, 1 species } \\
\hline Argyroneta aquatica (Clerck, 1758) & $\mathrm{SH}$ & TP & 27,86 \\
\hline \multicolumn{4}{|c|}{ DICTYNIDAE -7 genera, 13 species } \\
\hline $\begin{array}{l}\text { Archaeodictyna* } \text { ammophila }^{*, \mathrm{M}} \text { (Menge, } \\
\text { 1871) }\end{array}$ & $\mathrm{P}$ & WP & 106 \\
\hline $\begin{array}{l}\text { Archaeodictyna }^{*} \text { consecuta }^{* \mathrm{M}} \text { (O. Pickard- } \\
\text { Cambridge, } 1872)\end{array}$ & $\mathrm{P}$ & WP & 106 \\
\hline Argenna patula (Simon, 1874$)$ & SH & WP & 106 \\
\hline $\begin{array}{l}\text { Argenna subnigra (O. Pickard-Cambridge, } \\
\text { 1861) }\end{array}$ & $\mathrm{SH}$ & WP & 106 \\
\hline 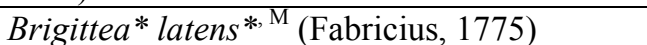 & SH & WP & 106 \\
\hline Devade tenella (Tyschchenko, 1965) & SA & WP & 106 \\
\hline Dictyna arundinacea (Linnaeus, 1758) & $\mathrm{P}$ & $\mathrm{TH}$ & 33,106 \\
\hline Dictyna major (Menge, 1868) & $\mathrm{P}$ & $\mathrm{TH}$ & 106 \\
\hline Dictyna pusilla* Thorell, 1856 & $\mathrm{~B}$ & TP & 106 \\
\hline $\begin{array}{l}\text { Dictyna ubsunurica* Marusik et Koponen, } \\
1998\end{array}$ & SA & $\mathrm{CP}$ & 106 \\
\hline Dictyna uncinata* Thorell, 1856 & $\mathrm{P}$ & TP & 56 \\
\hline Hackmania $^{*}$ prominula $^{* \mathrm{M}}$ (Tullgren, 1948) & B & $\mathrm{TH}$ & 56,106 \\
\hline $\begin{array}{l}\text { Mastigusa* macrophthalma*M (Kulczyński, } \\
\text { 1897) }\end{array}$ & $\mathrm{SH}$ & WP & 86 \\
\hline \multicolumn{4}{|c|}{ ERESIDAE -1 genus, 1 species } \\
\hline Eresus kollari Rossi, 1846 & SH & $\mathrm{TP}$ & $26,59,86,106$ \\
\hline \multicolumn{4}{|c|}{$\begin{array}{l}\text { EUTICHURIDAE }-1 \text { genus, } 4 \text { species } \\
\end{array}$} \\
\hline $\begin{array}{l}\text { Cheiracanthium* erraticum* (Walckenaer, } \\
\text { 1802) }\end{array}$ & $\mathrm{P}$ & $\mathrm{TP}$ & 106 \\
\hline $\begin{array}{l}\text { Cheiracanthium } * \text { oncognathum }{ }^{* \mathrm{M}} \text { Thorell, } \\
1871\end{array}$ & $\mathrm{P}$ & WP & 106 \\
\hline $\begin{array}{l}\text { Cheiracanthium* pennyi* O. Pickard- } \\
\text { Cambridge, } 1873\end{array}$ & $\mathrm{SH}$ & WP & 106 \\
\hline $\begin{array}{l}\text { Cheiracanthium } * \text { punctorium }^{* \mathrm{M}} \text { (Villers, } \\
1789 \text { ) }\end{array}$ & $\mathrm{SH}$ & WP & 106 \\
\hline \multicolumn{4}{|c|}{ GNAPHOSIDAE -12 genera, 45 species } \\
\hline Berlandina cinerea (Menge, 1868) & SA & WP & 106 \\
\hline Callilepis* nocturna* (Linnaeus, 1758$)$ & $\mathrm{P}$ & TP & $73,84,106$ \\
\hline Civizelotes* pygmaeus $*$ Miller, 1943 & SA & WP & 86,106 \\
\hline Drassodes* pubescens* $($ Thorell, 1856$)$ & SH & $\mathrm{TP}$ & $73,86,106$ \\
\hline Drassodes*villosus* (Thorell, 1856) & $\mathrm{SH}$ & TP & 56 \\
\hline Drassylus $*$ lutetianus $*$ (L. Koch, 1866$)$ & $\mathrm{SH}$ & WP & 86,106 \\
\hline Drassylus* pusillus* (C.L. Koch, 1833) & SH & TP & $86,106,113$ \\
\hline Gnaphosa inconspecta*, ${ }^{\mathrm{M}}$ Simon, 1878 & $\mathrm{P}$ & $\mathrm{TP}$ & 106 \\
\hline Gnaphosa jucunda ${ }^{* \mathrm{M}}$ Thorell, 1875 & SA & WP & 86 \\
\hline Gnaphosa leporina (L. Koch, 1866) & $\mathrm{P}$ & WP & $27,86,106,108$ \\
\hline Gnaphosa licent ${ }^{*}, \mathrm{M}$ Schenkel, 1953 & SA & $\mathrm{EP}$ & 113 \\
\hline Gnaphosa lucifuga (Walckenaer, 1802) & $\mathrm{P}$ & WP & 73 \\
\hline Gnaphosa montana (L. Koch, 1866) & $\mathrm{P}$ & WP & $15,73,98,105$ \\
\hline Gnaphosa muscorum* (L. Koch, 1866) & $\mathrm{P}$ & $\mathrm{TH}$ & 106 \\
\hline
\end{tabular}


Table 1 (continued). Таблица 1 (продолжение).

\begin{tabular}{|c|c|c|c|}
\hline Species & LAT & LON & Records in Novosibirsk Area \\
\hline \multicolumn{4}{|c|}{ GNAPHOSIDAE } \\
\hline $\begin{array}{l}\text { Gnaphosa saurica Ovtsharenko, Platnick et } \\
\text { Song, } 1992\end{array}$ & SA & WP & 105,106 \\
\hline $\begin{array}{l}\text { Gnaphosa tuvinica Marusik et Logunov, } \\
1992\end{array}$ & SA & $\mathrm{CP}$ & 106 \\
\hline $\begin{array}{l}\text { Gnaphosa ukrainica*,M Ovtsharenko, } \\
\text { Platnick et Song, } 1992\end{array}$ & SA & WP & 26,106 \\
\hline Haplodrassus cognatus* (Westring, 1861) & SH & $\mathrm{TP}$ & 86 \\
\hline $\begin{array}{l}\text { Haplodrassus minor } * \mathrm{M} \text { (O. Pickard- } \\
\text { Cambridge, 1879) }\end{array}$ & $\mathrm{P}$ & WP & 86 \\
\hline Haplodrassus moderatus* (Kulczyński, 1897 & $\mathrm{SH}$ & $\mathrm{TP}$ & 86 \\
\hline $\begin{array}{l}\text { Haplodrassus pseudosignifer* Marusik, } \\
\text { Hippa et Koponen, } 1996\end{array}$ & $\mathrm{SH}$ & WP & 106,113 \\
\hline Haplodrassus signifer (C.L. Koch, 1839) & $\mathrm{P}$ & TH & $49,73,86,106,113$ \\
\hline Haplodrassus soerensen ${ }^{*}($ Strand, 1900$)$ & SH & TP & 106 \\
\hline Haplodrassus umbratilis ${ }^{* \mathrm{M}}$ (L. Koch, 1866) & $\mathrm{SH}$ & WP & 73 \\
\hline Micaria dives (Lucas, 1846) & $\mathrm{P}$ & TP & 106 \\
\hline Micaria formicaria* (Sundevall, 1831) & $\mathrm{SH}$ & $\mathrm{TP}$ & 86,106 \\
\hline Micaria fulgens ${ }^{* \mathrm{M}}$ (Walckenaer, 1802) & SH & WP & 106 \\
\hline Micaria pulicaria* (Sundevall, 1831) & $\mathrm{P}$ & $\mathrm{TH}$ & 86,106 \\
\hline Micaria rossica Thorell, 1975 & $P$ & TH & 106 \\
\hline Micaria silesiaca* ${ }^{*}$ L. Koch, 1875 & $\mathrm{P}$ & WP & 86 \\
\hline Phaeocedus $^{*}$ braccatus $^{* \mathrm{M}}$ (L. Koch, 1866) & $\mathrm{SH}$ & $\mathrm{TP}$ & 86 \\
\hline Poecilochroa variana (C.L. Koch, 1839) & $\mathrm{P}$ & WP & 106 \\
\hline Urozelotes $*$ trifidus ${ }^{* \mathrm{M}}$ Tuneva, 2003 & SA & $\mathrm{CP}$ & 106 \\
\hline Zelotes apricorum* $($ L. Koch, 1876$)$ & $\mathrm{SH}$ & WP & 86,106 \\
\hline $\begin{array}{l}\text { Zelotes azsheganovae* Esyunin et Efimik, } \\
1992\end{array}$ & $\mathrm{SH}$ & WP & 86 \\
\hline Zelotes clivicola* $^{*}(\mathrm{~L}$. Koch, 1870) & SH & WP & 86,106 \\
\hline Zelotes electus $^{* \mathrm{M}}$ (C.L. Koch, 1839) & SH & WP & 86,106 \\
\hline Zelotes fratris ${ }^{*}{ }^{\mathrm{M}}$ Chamberlin, 1920 & $\mathrm{P}$ & EP & 86 \\
\hline Zelotes gallicus ${ }^{* \mathrm{M}}$ Simon, 1914 & SH & WP & 106 \\
\hline Zelotes latreille ${ }^{*}$ (Simon, 1878 ) & SH & WP & $53,80,86$ \\
\hline Zelotes longipes (L. Koch, 1866) & $\mathrm{P}$ & TP & $80,86,106$ \\
\hline Zelotes mundus (Kulczyński, 1897) & SA & WP & $86,106,113$ \\
\hline Zelotes petrensis* (C.L. Koch, 1839) & $\mathrm{P}$ & WP & 71 \\
\hline Zelotes puritanus ${ }^{* \mathrm{M}}$ Chamberlin, 1922 & $\mathrm{P}$ & TH & 74 \\
\hline Zelotes subterraneus (C.L. Koch, 1833) & SH & TP & 106 \\
\hline \multicolumn{4}{|c|}{ HAHNIIDAE -2 genera, 4 species } \\
\hline Antistea elegans (Blackwall, 1841) & SH & TP & 56,106 \\
\hline Hahnia nava* (Blackwall, 1841) & SH & $\mathrm{TP}$ & 106 \\
\hline Hahnia ononidum Simon, 1875 & SH & TH & 106 \\
\hline Hahnia pusilla* C.L. Koch, 1841 & $\mathrm{SH}$ & WP & 86 \\
\hline \multicolumn{4}{|c|}{ LINYPHIIDAE -50 genera, 82 species } \\
\hline Abacoproeces* saltuum* (L. Koch, 1872) & SH & WP & 86 \\
\hline Agyneta gulosa ${ }^{*, \mathrm{M}}(\mathrm{L} . \mathrm{Koch}, 1869)$ & SH & WP & 98,105 \\
\hline $\begin{array}{l}\text { Agyneta mollis (O. Pickard-Cambridge, } \\
1871 \text { ) }\end{array}$ & $\mathrm{B}$ & TP & 21 \\
\hline Agyneta ramosa Jackson, 1912 & $\mathrm{SH}$ & WP & 53,56 \\
\hline Agyneta rurestris*(C.L. Koch, 1836) & $\mathrm{P}$ & WP & $56,86,106$ \\
\hline Agyneta saaristoi Tanasevitch, 2000 & $\mathrm{~B}$ & $\mathrm{CP}$ & 78 \\
\hline
\end{tabular}


Table 1 (continued). Таблица 1 (продолжение).

\begin{tabular}{|c|c|c|c|}
\hline Species & LAT & LON & Records in Novosibirsk Area \\
\hline \multicolumn{4}{|c|}{ LINYPHIIDAE } \\
\hline Agyneta similis (Kulczyński, 1926) & $\mathrm{B}$ & TP & 78,112 \\
\hline Agyneta simplicitarsis* (Simon, 1884) & $\mathrm{SH}$ & WP & 86 \\
\hline $\begin{array}{l}\text { Agyneta subtilis (O. Pickard-Cambridge, } \\
\text { 1863) }\end{array}$ & $\mathrm{B}$ & $\mathrm{TP}$ & 21 \\
\hline Allomengea dentisetis ${ }^{*} \mathrm{M}$ (Grube, 1861) & $\mathrm{P}$ & EP & 53 \\
\hline Allomengea scopigera* (Grube, 1859) & $\mathrm{P}$ & TH & 53,56 \\
\hline Allomengea vidua (L. Koch, 1879) & $\mathrm{SH}$ & TH & 56,86 \\
\hline Anguliphantes cerinus (L. Koch, 1879) & $\mathrm{B}$ & $\mathrm{CP}$ & $21,56,79$ \\
\hline Araeoncus crassipes (Westring, 1861) & SH & WP & 106 \\
\hline Araeoncus vorkutensis Tanasevitch, 1984 & $\mathrm{~B}$ & $\mathrm{CP}$ & 79 \\
\hline $\begin{array}{l}\text { Archaraeoncus }^{*} \text { prospiciens }^{*, \mathrm{M}} \text { (Thorell, } \\
1875 \text { ) }\end{array}$ & $\mathrm{SH}$ & WP & 79 \\
\hline Bathyphantes eumenis (L. Koch, 1879) & $\mathrm{B}$ & TH & 21 \\
\hline Bathyphantes gracilis (Blackwall, 1841) & $\mathrm{P}$ & TH & 56 \\
\hline Bathyphantes nigrinus (Westring, 1851) & SH & WP & 56 \\
\hline Bathyphantes parvulus (Westring, 1851) & $\mathrm{B}$ & TP & $35,56,86$ \\
\hline Bolyphantes alticeps (Sundevall, 1833) & $\mathrm{SH}$ & TP & 53,56 \\
\hline Centromerita $*$ bicolor $^{*, \mathrm{M}}$ (Blackwall, 1833) & $\mathrm{SH}$ & TH & 53 \\
\hline $\begin{array}{l}\text { Centromerus arcanus (O. Pickard- } \\
\text { Cambridge, 1873) }\end{array}$ & B & WP & 21 \\
\hline Centromerus clarus (L. Koch, 1879) & $\mathrm{B}$ & TP & $21,53,56$ \\
\hline Centromerus incilium (L. Koch, 1881) & $\mathrm{B}$ & WP & 21 \\
\hline Centromerus sylvaticus (Blackwall, 1841) & $\mathrm{P}$ & $\mathrm{TH}$ & 56,64 \\
\hline Ceratinella brevis (Wider, 1834) & $\mathrm{P}$ & TP & 21,106 \\
\hline Decipiphantes decipiens (L. Koch, 1879) & $\mathrm{B}$ & WP & 21 \\
\hline Dicymbium nigrum (Blackwall, 1834) & $\mathrm{P}$ & WP & 56 \\
\hline Dicymbium tibiale ${ }^{* \mathrm{M}}$ (Blackwall, 1836) & $\mathrm{P}$ & WP & 53 \\
\hline Diplostyla concolor (Wider, 1834) & $\mathrm{B}$ & TH & $21,50,53,56,86$ \\
\hline Drapetisca socialis (Sundevall, 1833) & $\mathrm{P}$ & TP & 51,56 \\
\hline Erigone $^{*}$ atra $*$ Blackwall, 1833 & $\mathrm{P}$ & TH & 106 \\
\hline Erigone $^{*}$ dentipalpis $*$ (Wider, 1834) & $\mathrm{P}$ & TP & $98,105,106$ \\
\hline Erigonoplus $^{*}$ globipes $^{*}{ }^{\mathrm{M}}$ (L. Koch, 1872) & SA & WP & 106 \\
\hline $\begin{array}{l}\text { Erigonoplus } * \text { spinifemuralis }{ }^{*, \mathrm{M}} \text { Dimitrov, } \\
2003\end{array}$ & SA & WP & 106 \\
\hline Floronia bucculenta (Clerck, 1758) & $\mathrm{P}$ & TP & $51,52,64$ \\
\hline Gnathonarium dentatum (Wider, 1834) & $\mathrm{P}$ & TP & 106 \\
\hline Gonatium rubellum (Blackwall, 1841) & $\mathrm{B}$ & TP & 21,86 \\
\hline Gonatium rubens* (Blackwall, 1833) & $\mathrm{P}$ & TP & 86 \\
\hline Gongylidium rufipes (Linnaeus, 1758) & $\mathrm{P}$ & WP & 56,86 \\
\hline Helophora insignis (Blackwall, 1841) & $\mathrm{P}$ & TH & $51,56,64$ \\
\hline Lasiargus $^{*}$ hirsutus $^{*}, \mathrm{M}$ (Menge, 1869) & SH & TP & 86 \\
\hline Leptorhoptrum $*$ robustum $*$ (Westring, 1851$)$ & SH & TH & 86 \\
\hline Linyphia hortensis* Sundevall, 1830 & $\mathrm{P}$ & TP & 40,64 \\
\hline Linyphia triangularis (Clerck, 1758) & $\mathrm{P}$ & TH & $51,53,56,59,100$ \\
\hline Lophomma punctatum (Blackwall, 1842) & $\mathrm{SH}$ & WP & 86 \\
\hline $\begin{array}{l}\text { Macrargus multesimus (O. Pickard- } \\
\text { Cambridge, 1875) }\end{array}$ & B & $\mathrm{TH}$ & 21 \\
\hline Micrargus* herbigradus* (Blackwall, 1854) & $\mathrm{P}$ & TP & 53 \\
\hline Microlinyphia* pusilla* (Sundevall, 1830) & $\mathrm{P}$ & TH & 86,106 \\
\hline Microneta viaria (Blackwall, 1841) & $\mathrm{P}$ & $\mathrm{TH}$ & 56 \\
\hline Moebelia $^{*}$ penicillata ${ }^{*, \mathrm{M}}$ (Westring, 1851) & $\mathrm{P}$ & WP & 57 \\
\hline
\end{tabular}


Table 1 (continued). Таблица 1 (продолжение).

\begin{tabular}{|c|c|c|c|}
\hline Species & LAT & LON & Records in Novosibirsk Area \\
\hline \multicolumn{4}{|c|}{ LINYPHIIDAE } \\
\hline Neriene clathrata* (Sundevall, 1830) & SH & $\mathrm{TH}$ & 86 \\
\hline Neriene emphana (Walckenaer, 1841) & $\mathrm{SH}$ & TP & $56,59,79$ \\
\hline Neriene montana $*($ Clerck, 1758$)$ & $\mathrm{SH}$ & TP & $13,56,86$ \\
\hline Neriene peltata* $($ Wider, 1834) & $\mathrm{P}$ & WP & 86 \\
\hline Neriene radiata* (Walckenaer, 1841) & $\mathrm{SH}$ & $\mathrm{TH}$ & 56,106 \\
\hline Oedothorax* apicatus* (Blackwall, 1850) & SA & WP & $98,105,106$ \\
\hline Oryphantes geminus (Tanasevitch, 1982) & $\mathrm{B}$ & WP & 21 \\
\hline $\begin{array}{l}\text { Panamomops dybowskii (O. Pickard- } \\
\text { Cambridge, 1873) }\end{array}$ & $\mathrm{B}$ & $\mathrm{CP}$ & 21,79 \\
\hline Pocadicnemis* pumila* (Blackwall, 1841) & $\mathrm{P}$ & $\mathrm{TH}$ & 53 \\
\hline $\begin{array}{l}\text { Sauron* fissocornis }{ }^{*}{ }^{\mathrm{M}} \text { Eskov in Eskov et } \\
\text { Marusik, } 1995\end{array}$ & $\mathrm{SH}$ & $\mathrm{CP}$ & 86 \\
\hline Semljicola angulatus (Holm, 1963) & $\mathrm{B}$ & TP & 79 \\
\hline $\begin{array}{l}\text { Silometopus * ambiguus* (O. Pickard- } \\
\text { Cambridge, } 1906)\end{array}$ & $\mathrm{SH}$ & WP & 106 \\
\hline Silometopus $*$ reussi $*$ (Thorell, 1871) & $\mathrm{SH}$ & TP & 106 \\
\hline Stemonyphantes $*$ lineatus $*$ (Linnaeus, 1758) & $\mathrm{SH}$ & WP & 86,106 \\
\hline $\begin{array}{l}\text { Stemonyphantes } * \text { taiganus } * \text { (Ermolajev, } \\
1834 \text { ) }\end{array}$ & $\mathrm{B}$ & $\mathrm{CP}$ & 79,78 \\
\hline Styloctetor stativus* (Simon, 1881) & $\mathrm{SH}$ & $\mathrm{TH}$ & 86 \\
\hline $\begin{array}{l}\text { Tapinocyba ? pallens (O. Pickard- } \\
\text { Cambridge, 1873) }\end{array}$ & $\mathrm{SH}$ & WP & 106 \\
\hline Tenuiphantes nigriventris (L. Koch, 1879) & $\mathrm{B}$ & TP & 53,79 \\
\hline Tenuiphantes tenebricola (Wider, 1834) & $\mathrm{SH}$ & WP & 56 \\
\hline Thyreosthenius parasiticus (Westring, 1851) & $\mathrm{P}$ & TH & 56 \\
\hline Trichoncus vasconicus Denis, 1944 & $\mathrm{SH}$ & WP & 86,106 \\
\hline $\begin{array}{l}\text { Trichopterna* cito* (O. Pickard-Cambridge, } \\
\text { 1872) }\end{array}$ & SA & WP & 86,106 \\
\hline Uralophantes $*$ troitskensis ${ }^{*, \mathrm{M}}$ Esyunin, 1992 & SA & $\mathrm{CP}$ & 86 \\
\hline Walckenaeria antica (Wider, 1834) & $\mathrm{B}$ & WP & 21,53 \\
\hline $\begin{array}{l}\text { Walckenaeria atrotibialis } * \text { O. Pickard- } \\
\text { Cambridge, } 1878\end{array}$ & $\mathrm{P}$ & $\mathrm{TH}$ & 53,86 \\
\hline $\begin{array}{l}\text { Walckenaeria kochi* (O. Pickard-Cambridge, } \\
\text { 1873) }\end{array}$ & $\mathrm{SH}$ & $\mathrm{TH}$ & 56 \\
\hline Walckenaeria nudipalpis* (Westring, 1851) & $\mathrm{P}$ & TP & 53 \\
\hline $\begin{array}{l}\text { Walckenaeria unicornis* O. Pickard- } \\
\text { Cambridge, } 1861\end{array}$ & $\mathrm{P}$ & WP & 53 \\
\hline Walckenaerianus esyunini Tanasevitch, 2004 & SA & WP & 106 \\
\hline Zornella cultrigera (L. Koch, 1879) & $\mathrm{B}$ & TP & 21,106 \\
\hline \multicolumn{4}{|c|}{ LIOCRANIDAE -1 genus, 6 species } \\
\hline Agroeca brunnea (Blackwall, 1833) & $\mathrm{P}$ & TP & 56 \\
\hline Agroeca cuprea Menge, 1873 & $\mathrm{SH}$ & WP & 56 \\
\hline Agroeca dentigera ${ }^{*}, \mathrm{M}$ Kulczyński, 1913 & $\mathrm{SH}$ & WP & 86,106 \\
\hline Agroeca lusatica* (L. Koch, 1875) & $\mathrm{SH}$ & WP & 86 \\
\hline Agroeca maculata* L. Koch, 1879 & SH & TP & 86 \\
\hline Agroeca ornata ${ }^{*, \mathrm{M}}$ Banks, 1892 & $\mathrm{SH}$ & EP & 56 \\
\hline \multicolumn{4}{|c|}{ LYCOSIDAE - 12 genera, 52 species } \\
\hline Acantholycosa lignaria (Clerck, 1758) & $\mathrm{SH}$ & TP & 58 \\
\hline Acantholycosa norvegica (L. Koch, 1875) & $\mathrm{B}$ & TP & 21,58 \\
\hline Allohogna singoriensis (Laxman, 1770) & SA & WP & $\begin{array}{l}11,25,27,28,29,34,49,84,86,89,92, \\
106,111\end{array}$ \\
\hline
\end{tabular}


Table 1 (continued). Таблица 1 (продолжение).

\begin{tabular}{|c|c|c|c|}
\hline Species & LAT & LON & Records in Novosibirsk Area \\
\hline \multicolumn{4}{|c|}{ LYCOSIDAE } \\
\hline Alopecosa cf. albostriata & SH & EP & 106 \\
\hline Alopecosa azsheganovae Esyunin, 1996 & SH & $\mathrm{CP}$ & 86 \\
\hline Alopecosa cuneata (Clerck, 1758) & $\mathrm{SH}$ & TP & $17,18,50,86,106,113$ \\
\hline Alopecosa cursor (Hahn, 1831) & $\mathrm{P}$ & WP & $56,88,98,106$ \\
\hline Alopecosa fabrilis (Clerck, 1758) & SH & TP & $18,29,56$ \\
\hline Alopecosa farinosa (Herman, 1879) & SH & TP & $17,49,86,106,113$ \\
\hline Alopecosa kasakhstanica* Savelyeva, 1972 & $\mathrm{SH}$ & $\mathrm{CP}$ & $56,65,69$ \\
\hline $\begin{array}{l}\text { Alopecosa kovblyuki*, Madolny et } \\
\text { Ponomarev, } 2012\end{array}$ & SA & WP & 106 \\
\hline Alopecosa pinetorum Thorell, 1856 & SH & WP & 56 \\
\hline Alopecosa pulverulenta (Clerck, 1758) & SH & TP & $18,29,49,46,56,71,86,99$ \\
\hline Alopecosa schmidti (Hahn, 1835) & SH & WP & $7,86,106,113$ \\
\hline Alopecosa sulzeri (Pavesi, 1863) & SA & WP & 86,106 \\
\hline Alopecosa taeniata (C.L. Koch, 1835) & SH & WP & 106 \\
\hline Alopecosa taeniopus (Kulczyński, 1895) & SA & WP & 106 \\
\hline Arctosa $*$ alpigena $*$ (Doleschall, 1852) & $\mathrm{P}$ & TH & 106 \\
\hline Arctosa* leopardus* (Sundevall, 1833) & SH & WP & 106 \\
\hline Arctosa* stigmosa* (Thorell, 1875) & $\mathrm{P}$ & $\mathrm{TP}$ & 49 \\
\hline Evippa sp. & $\mathrm{E}$ & $\mathrm{E}$ & 106 \\
\hline Hygrolycosa $^{*}$ rubrofasciata* $($ Ohlert, 1865$)$ & $\mathrm{B}$ & WP & 14 \\
\hline Mustelicosa dimidiata (Thorell, 1875) & SA & WP & 106 \\
\hline Pardosa agrestis (Westring, 1861) & SH & TP & $17,18,29,56,86,106,111,113$ \\
\hline Pardosa agricola (Thorell, 1856) & SH & WP & $18,45,46,56,99,111$ \\
\hline Pardosa amentata (Clerck, 1758) & $\mathrm{P}$ & WP & 56,79 \\
\hline Pardosa bifasciata (C.L. Koch, 1836) & $\mathrm{SH}$ & WP & $17,86,106,113$ \\
\hline Pardosa eiseni $*$ (Thorell, 1875) & $\mathrm{B}$ & TP & 86 \\
\hline Pardosa fulvipes (Collett, 1875) & $\mathrm{SH}$ & WP & $6,18,75,86,106$ \\
\hline Pardosa italica Tongiorgi, 1966 & $\mathrm{P}$ & WP & 106 \\
\hline Pardosa luctinosa* Simon, 1876 & SA & WP & 106 \\
\hline Pardosa lugubris (Walckenaer, 1802) & SH & WP & $18,23,21,45,46,56,86,99,106,111$ \\
\hline Pardosa maisa* Hippa et Mannila, 1982 & $\mathrm{SH}$ & WP & 86 \\
\hline $\begin{array}{l}\text { Pardosa masurae }{ }^{*} \text { M Esyunin et Efimik, } \\
1998\end{array}$ & SH & $\mathrm{CP}$ & 86 \\
\hline ? Pardosa monticola (Clerck, 1758) & SH & WP & $17,18,56,88,99,111$ \\
\hline Pardosa paludicola (Clerck, 1758) & SH & TP & $45,56,86,106$ \\
\hline Pardosa palustris (Linnaeus, 1758) & $\mathrm{P}$ & $\mathrm{TH}$ & $6,17,18,22,23,29,45,50,46,56,99,111$ \\
\hline Pardosa plumipes (Thorell, 1875) & SH & $\mathrm{TP}$ & $\begin{array}{l}6,7,17,18,29,45,46,56,58,86,88,98 \\
99,105,106,111\end{array}$ \\
\hline Pardosa prativaga (L. Koch, 1870) & SH & WP & $6,17,18,46,56,99$ \\
\hline Pardosa proxima (C.L. Koch, 1847) & SH & TP & $29,30,107$ \\
\hline Pardosa pullata (Clerck, 1758) & SH & WP & 17,75 \\
\hline Pardosa riparia (C.L. Koch, 1847) & SH & $\mathrm{TP}$ & 17 \\
\hline Pardosa schenkeli* Lessert, 1904 & SH & TP & 86,106 \\
\hline Pirata piraticus (Clerck, 1758) & SH & TH & $13,29,56,86,106$ \\
\hline Piratula hygrophila (Thorell, 1872) & $\mathrm{P}$ & $\mathrm{TP}$ & $49,56,79$ \\
\hline Piratula uliginosa (Thorell, 1856) & SH & WP & 86 \\
\hline Trochosa robusta (Simon, 1876) & $\mathrm{P}$ & TP & 86,106 \\
\hline Trochosa ruricola (De Geer, 1778) & $\mathrm{SH}$ & $\mathrm{TH}$ & $10,17,27,49,46,56,86,99,106$ \\
\hline $\begin{array}{l}\text { Trochosa spinipalpis (F.O. Pickard- } \\
\text { Cambridge, 1895) }\end{array}$ & B & $\mathrm{TP}$ & $11,21,56,86$ \\
\hline
\end{tabular}


Table 1 (continued).

Таблица 1 (продолжение).

\begin{tabular}{|c|c|c|c|}
\hline Species & LAT & LON & Records in Novosibirsk Area \\
\hline \multicolumn{4}{|c|}{ LYCOSIDAE } \\
\hline Trochosa terricola Thorell, 1856 & $\mathrm{P}$ & TH & $6,21,49,56,66,86,106$ \\
\hline Xerolycosa miniata (C.L. Koch, 1834) & SH & WP & $29,49,46,56,86,99,105,106,111$ \\
\hline Xerolycosa nemoralis (Westring, 1861) & SH & TP & $6,29,46,56,98,99,106,111$ \\
\hline \multicolumn{4}{|c|}{ MIMETIDAE -1 genus, 2 species } \\
\hline Ero cambridgei ${ }^{*}, \mathrm{M}$ Kulczyński, 1911 & $\mathrm{~B}$ & TP & 86 \\
\hline Ero furcata Villers, 1789) & SH & TP & 56 \\
\hline \multicolumn{4}{|c|}{ MITURGIDAE - 1 genus, 3 species } \\
\hline 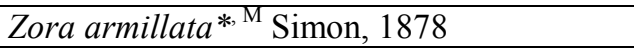 & $\mathrm{SH}$ & WP & 86 \\
\hline Zora nemoralis* (Blackwall, 1861) & SH & TP & 86 \\
\hline Zora spinimana (Dufour, 1820) & SH & TP & $56,86,106$ \\
\hline \multicolumn{4}{|c|}{ OXYOPIDAE - 1 genus, 1 species } \\
\hline Oxyopes xinjiangensis $\mathrm{Hu}$ et $\mathrm{Wu}, 1989$ & $\mathrm{SA}$ & $\mathrm{CP}$ & 106 \\
\hline \multicolumn{4}{|c|}{ PHILODROMIDAE -4 genera, 15 species } \\
\hline Philodromus aureolus* (Clerck, 1758) & $\mathrm{P}$ & $\mathrm{TP}$ & 108 \\
\hline Philodromus cespitum* (Walckenaer, 1802) & $\mathrm{P}$ & TH & $74,86,106$ \\
\hline Philodromus utotchkini Marusik, 1991 & $\mathrm{~B}$ & EP & 21 \\
\hline Rhysodromus fallax (Sundevall, 1833) & $\mathrm{P}$ & TP & 106,108 \\
\hline Rhysodromus histrio (Latreille, 1819) & SH & TH & $28,36,43,81,82,83,84,86,106,110$ \\
\hline Thanatus arenarius L. Koch, 1872 & SA & WP & $43,56,74,84,86,106,108$ \\
\hline Thanatus atratus Simon, 1875 & SH & WP & $84,86,106$ \\
\hline Thanatus formicinus (Clerck, 1758) & $\mathrm{SH}$ & $\mathrm{TH}$ & $39,53,86$ \\
\hline Thanatus mikhailovi* Logunov, 1996 & SA & $\mathrm{CP}$ & 106 \\
\hline Thanatus pictus L. Koch, 1881 & SH & WP & 106 \\
\hline Thanatus sabulosus (Menge, 1875) & $\mathrm{SH}$ & TP & 56 \\
\hline Thanatus striatus C.L. Koch, 1845 & $\mathrm{P}$ & $\mathrm{TH}$ & 86 \\
\hline Tibellus macellus* Simon, 1875 & SH & TP & 86 \\
\hline Tibellus maritimus (Menge, 1875) & $\mathrm{P}$ & TH & $4,33,56,86,113$ \\
\hline Tibellus oblongus (Walckenaer, 1802) & $\mathrm{P}$ & $\mathrm{TH}$ & $4,39,56,86,106,108$ \\
\hline \multicolumn{4}{|c|}{ PHOLCIDAE* -1 genus, 1 species } \\
\hline Pholcus $^{*}$ opilionoides $*, \mathrm{M}$ (Schrank, 1871) & $\mathrm{P}$ & WP & 56 \\
\hline \multicolumn{4}{|c|}{ PHRUROLITHIDAE -1 genus, 1 species } \\
\hline Phrurolithus festivus (C.L. Koch, 1835) & SH & TP & 56 \\
\hline \multicolumn{4}{|c|}{ PISAURIDAE -2 genera, 2 species } \\
\hline Dolomedes plantarius (Clerck, 1758) & $\mathrm{SH}$ & WP & 56 \\
\hline Pisaura* mirabilis* $($ Clerck, 1758$)$ & SH & WP & 86,106 \\
\hline \multicolumn{4}{|c|}{ SALTICIDAE - 19 genera, 36 species } \\
\hline Aelurillus v-insignitus (Clerck, 1758) & $\mathrm{P}$ & TP & $86,98,105,106$ \\
\hline Asianellus festivus (C.L. Koch, 1834) & $\mathrm{SH}$ & TP & $43,56,77,86,103,101,106$ \\
\hline $\begin{array}{l}\text { Asianellus kazakhstanicus Logunov et } \\
\text { Heçiak, } 1996\end{array}$ & $\mathrm{E}$ & $\mathrm{E}$ & 106 \\
\hline Attulus* distinguendus* (Simon, 1868) & $\mathrm{P}$ & TP & 106 \\
\hline $\begin{array}{l}\text { Attulus* saltator (O. Pickard-Cambridge, } \\
1868)\end{array}$ & SA & WP & 86,106 \\
\hline $\begin{array}{l}\text { Chalcoscirtus brevicymbialis Wunderlich, } \\
1980\end{array}$ & $\mathrm{SH}$ & WP & 106 \\
\hline Chalcoscirtus nigritus (Thorell, 1875) & SH & WP & 106 \\
\hline Euophrys frontalis (Walckenaer, 1802) & $\mathrm{P}$ & $\mathrm{TP}$ & $56,78,106$ \\
\hline Evarcha arcuata (Clerck, 1758) & $\mathrm{P}$ & $\mathrm{TP}$ & $\begin{array}{l}4,31,32,39,47,56,86,87,103,106,108, \\
112\end{array}$ \\
\hline Evarcha falcata (Clerck, 1758) & $\mathrm{P}$ & WP & $\begin{array}{l}6,5,4,16,15,14,22,31,32,39,40,43,47, \\
56,71,86,95,102,100,106,108,112\end{array}$ \\
\hline
\end{tabular}


Table 1 (continued). Таблица 1 (продолжение).

\begin{tabular}{|c|c|c|c|}
\hline Species & LAT & LON & Records in Novosibirsk Area \\
\hline \multicolumn{4}{|c|}{ SALTICIDAE } \\
\hline Evarcha laetabunda (C.L. Koch, 1846) & $\mathrm{P}$ & TP & $47,86,106,112$ \\
\hline Evarcha michailovi Logunov, 1992 & $\mathrm{SH}$ & WP & $42,68,73,86,92,90,106,112$ \\
\hline Heliophanus auratus C.L. Koch, 1835 & $\mathrm{P}$ & WP & $16,42,56,57,86,87,106,112$ \\
\hline Heliophanus dubius ${ }^{*}$ C.L. Koch, 1835 & $\mathrm{P}$ & TP & 56 \\
\hline Heliophanus flavipes (Hahn, 1832) & $\mathrm{P}$ & TP & $16,32,42,43,56,73,86,106$ \\
\hline Heliophanus patagiatus Thorell, 1875 & $\mathrm{P}$ & $\mathrm{TP}$ & 56 \\
\hline Heliophanus ussuricus Kulczyński, 1895 & $\mathrm{SH}$ & EP & 73 \\
\hline Marpissa pomatia (Walckenaer, 1802) & $\mathrm{P}$ & $\mathrm{TP}$ & $4,31,39,40,56,57,77,87,100,112$ \\
\hline Marpissa radiata (Grube, 1859$)$ & $\mathrm{P}$ & WP & $4,12,36,39,56,86,106$ \\
\hline Neon $*$ rayi $^{*{ }^{\mathrm{M}}}$ (Simon, 1875$)$ & SH & WP & 106 \\
\hline Pellenes albopilosus (Tyschchenko, 1965) & SA & $\mathrm{CP}$ & 106 \\
\hline $\begin{array}{l}\text { Pellenes epularis* (O. Pickard-Cambridge, } \\
1872)\end{array}$ & $\mathrm{SH}$ & WP & 106 \\
\hline Pellenes sibiricus Logunov et Marusik, 1994 & SH & EP & 106 \\
\hline Phlegra fasciata (Hahn, 1826) & $\mathrm{P}$ & TP & $73,86,106$ \\
\hline Pseudeuophrys erratica (Walckenaer, 1826) & $\mathrm{P}$ & TP & $53,56,79$ \\
\hline Pseudeuophrys obsoleta (Simon, 1868) & SH & TP & 102 \\
\hline Salticus cingulatus (Panzer, 1797) & $\mathrm{P}$ & TP & 106 \\
\hline Sibianor aurocinctus (Ohlert, 1865) & $\mathrm{P}$ & WP & 4 \\
\hline Sibianor larae Logunov, 2001 & $\mathrm{P}$ & EP & 86 \\
\hline Sitticus terebratus* (Clerck, 1758) & $\mathrm{P}$ & TP & 56 \\
\hline $\begin{array}{l}\text { Sittiflor* inexpectus Logunov et Kronestedt, } \\
1997\end{array}$ & $\mathrm{SH}$ & WP & 32,56 \\
\hline Sittiflor* floricola (C.L. Koch, 1837) & $\mathrm{P}$ & TP & 39,56 \\
\hline 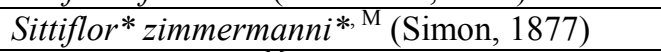 & SH & WP & 106 \\
\hline $\begin{array}{l}\text { Sittisax* ranieri }{ }^{*, \mathrm{M}} \text { (Peckham et Peckham, } \\
1909)\end{array}$ & $\mathrm{B}$ & $\mathrm{TH}$ & 86 \\
\hline Synageles venator (Lucas, 1836) & $\mathrm{P}$ & TH & $4,16,47$ \\
\hline Talavera aperta (Miller, 1971) & SH & WP & 112 \\
\hline \multicolumn{4}{|c|}{ SPARASSIDAE $*-1$ genus, 1 species } \\
\hline Micrommata $*$ virescens $*($ Clerck, 1758$)$ & SH & $\mathrm{TP}$ & 56,106 \\
\hline \multicolumn{4}{|c|}{ TETRAGNATHIDAE -3 genera, 9 species } \\
\hline Metellina* mengei* (Blackwall, 1869) & SH & WP & 56 \\
\hline Pachygnatha clercki Sundevall, 1823 & $\mathrm{P}$ & $\mathrm{TH}$ & $26,40,56,86,106$ \\
\hline Pachygnatha degeeri $*$ Sundevall, 1830 & SH & TP & 86,106 \\
\hline Pachygnatha listeri Sundevall, 1830 & $\mathrm{~B}$ & TP & $21,40,56,86,106$ \\
\hline Tetragnatha* dearmata* Thorell, 1873 & SH & $\mathrm{TH}$ & 56 \\
\hline Tetragnatha* extensa* (Linnaeus, 1758$)$ & $\mathrm{P}$ & TH & 86 \\
\hline Tetragnatha* montana* Simon, 1874 & SH & TP & 56 \\
\hline Tetragnatha* obtusa* C.L. Koch, 1837 & SH & TP & 86 \\
\hline Tetragnatha* pinicola $*$ L. Koch, 1870 & SH & $\mathrm{TP}$ & 56,106 \\
\hline \multicolumn{4}{|c|}{ THERIDIIDAE -11 genera, 16 species } \\
\hline Asagena* phalerata*(Panzer, 1801) & $\mathrm{SH}$ & TP & 86,106 \\
\hline Cryptachaea* ${ }^{*}$ riparia* (Blackwall, 1834) & SH & $\mathrm{TP}$ & 56 \\
\hline Enoplognatha* ${ }^{*}$ ovata ${ }^{* \mathrm{M}}($ Clerck, 1758) & SH & WP & 56 \\
\hline Euryopis $^{*}$ flavomaculata $^{*}($ C.L. Koch, 1836$)$ & SH & $\mathrm{TP}$ & 86,106 \\
\hline 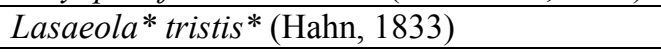 & $\mathrm{SH}$ & WP & 106 \\
\hline Neottiura* bimaculata* (Linnaeus, 1767 ) & SH & TH & $56,86,112$ \\
\hline $\begin{array}{l}\text { Parasteatoda* tepidariorum* (C.L. Koch, } \\
\text { 1841) }\end{array}$ & $\mathrm{P}$ & TH & 100 \\
\hline
\end{tabular}


Table 1 (continued). Таблица 1 (продолжение).

\begin{tabular}{|c|c|c|c|}
\hline Species & LAT & LON & Records in Novosibirsk Area \\
\hline \multicolumn{4}{|c|}{ THERIDIIDAE } \\
\hline Phylloneta* impressa* L. Koch, 1881 & $\mathrm{P}$ & $\mathrm{TH}$ & 112 \\
\hline $\begin{array}{l}\text { Robertus arundineti* (O. Pickard- } \\
\text { Cambridge, } 1871)\end{array}$ & $\mathrm{P}$ & WP & 113 \\
\hline Robertus heydemanni Wiehle, 1965 & SH & WP & 36,54 \\
\hline Steatoda albomaculata (De Geer, 1778) & $\mathrm{P}$ & TH & 106 \\
\hline Steatoda bipunctata $*$ (Linnaeus, 1758$)$ & $\mathrm{SH}$ & TH & $14,56,106,108$ \\
\hline Steatoda castanea ${ }^{* \mathrm{M}}$ (Clerck, 1758) & $\mathrm{P}$ & TH & 56 \\
\hline Steatoda grossa* (C.L. Koch, 1838) & $\mathrm{P}$ & TH & 16,56 \\
\hline Theridion* ${ }^{\text {innocuum }}{ }^{* \mathrm{M}}$ Thorell, 1875 & SH & $\mathrm{CP}$ & $106,110,112$ \\
\hline Theridion* varians* Hahn, 1833 & SH & TH & 56,106 \\
\hline \multicolumn{4}{|c|}{ THOMISIDAE -6 genera, 21 species } \\
\hline Coriarachne $^{*}$ depressa $^{*}$ (C.L. Koch, 1837) & SH & TP & 66 \\
\hline Heriaeus horridus Tyschchenko, 1965 & SA & WP & 106 \\
\hline Heriaeus mellottee ${ }^{*}$ Simon, 1886 & SH & EP & 86,106 \\
\hline Misumena $*$ vatia $*($ Clerck, 1758$)$ & $\mathrm{P}$ & TH & 56,60 \\
\hline Ozyptila praticola (C.L. Koch, 1837) & $\mathrm{P}$ & TP & $21,56,86$ \\
\hline Ozyptila rauda* Simon, 1875 & SH & WP & 106 \\
\hline Ozyptila scabricula (Westring, 1851) & SH & TP & 86,106 \\
\hline Ozyptila trux (Blackwall, 1846) & SH & TH & $56,86,106$ \\
\hline Tmarus piger (Walckenaer, 1802) & SH & TP & 56 \\
\hline Xysticus audax (Schrank, 1803) & $\mathrm{SH}$ & TP & $15,39,63$ \\
\hline Xysticus cristatus (Clerck, 1758) & $\mathrm{SH}$ & WP & $\begin{array}{l}24,28,41,42,43,53,47,56, \\
72,84,86,92,95,106,108, \\
112,113\end{array}$ \\
\hline Xysticus lanio* C.L. Koch, 1835 & $\mathrm{SH}$ & WP & 53 \\
\hline Xysticus luctuosus* (Blackwall, 1846) & SH & TH & $4,39,53,56$ \\
\hline Xysticus ninnii* Thorell, 1872 & SH & WP & $4,10,32,43,56,85,86,98,105,106,109$ \\
\hline $\begin{array}{l}\text { Xysticus pseudocristatus Azarkina et } \\
\text { Logunov, } 2001\end{array}$ & SA & $\mathrm{CP}$ & 54 \\
\hline Xysticus robustus* (Hahn, 1832) & $\mathrm{SH}$ & WP & 86,106 \\
\hline Xysticus sabulosus ${ }^{* \mathrm{M}}$ (Hahn, 1832) & $\mathrm{SH}$ & WP & 86 \\
\hline Xysticus striatipes ${ }^{*}$ L. Koch, 1870 & SH & WP & $33,43,86,106$ \\
\hline Xysticus sp. & $\mathrm{E}$ & $\mathrm{E}$ & 106 \\
\hline Xysticus ulmi* (Hahn, 1831) & $\mathrm{SH}$ & WP & 56,86 \\
\hline Xysticus viduus Kulczyński, 1898 & $\mathrm{SH}$ & WP & $10,28,37,86,106$ \\
\hline \multicolumn{4}{|c|}{ TITANOECIDAE -1 genus, 3 species } \\
\hline Titanoeca ${ }^{*}$ nivalis ${ }^{*{ }^{\mathrm{M}}}$ Simon, 1874 & $\mathrm{~B}$ & TH & 106 \\
\hline Titanoeca $*$ quadriguttata ${ }^{*}{ }^{\mathrm{M}}$ Hahn, 1833 & SH & WP & 86,106 \\
\hline Titanoeca* schineri $^{*}$ L. Koch, 1872 & $\mathrm{P}$ & WP & 86,106 \\
\hline \multicolumn{4}{|c|}{ ULOBORIDAE* - 1 genus, 1 species } \\
\hline Uloborus* walckenaerius* Latreille, 1806 & SH & TP & 106 \\
\hline
\end{tabular}

Abbreviations: * — the first record for Novisibirsk Area; M — the first record for West Siberian Plain (area 'M' in [Mikhailov, 2013]). LAT - latitudinal components of the range: B — boreal species, SH — subboreal humid species, SA - subarid species, $\mathrm{P}$ polyzonal species, E - endemic; LON - latitudinal components of the range: TH - trans-Holarctic species, TP - trans-Palaearctic species, WP — west-Palaearctic species, CP — central-Palaearctic species, EP — east-Palaearctic species. 1-113 — collecting localities, for their decoding see Map.

Обозначения: * - таксон впервые приводится для Новосибирской области; М - вид впервые приводится для равнинной части Западной Сибири (регион М по: [Михайлов, 2013]). LAT — широтная компонента ареала: В — бореальный вид, SH суббореальный гумидный, SA - субаридный, P — полизональный, E — эндемик; LON — долготная компонента ареала: ТН трансголарктический, ТP — транспалеарктический, WP - западнопалеарктический, СР — центральнопалеарктический, ЕР — восточнопалеарктический. 1-113 - точки сбора: расшифровку см. в подписи к Карте. 


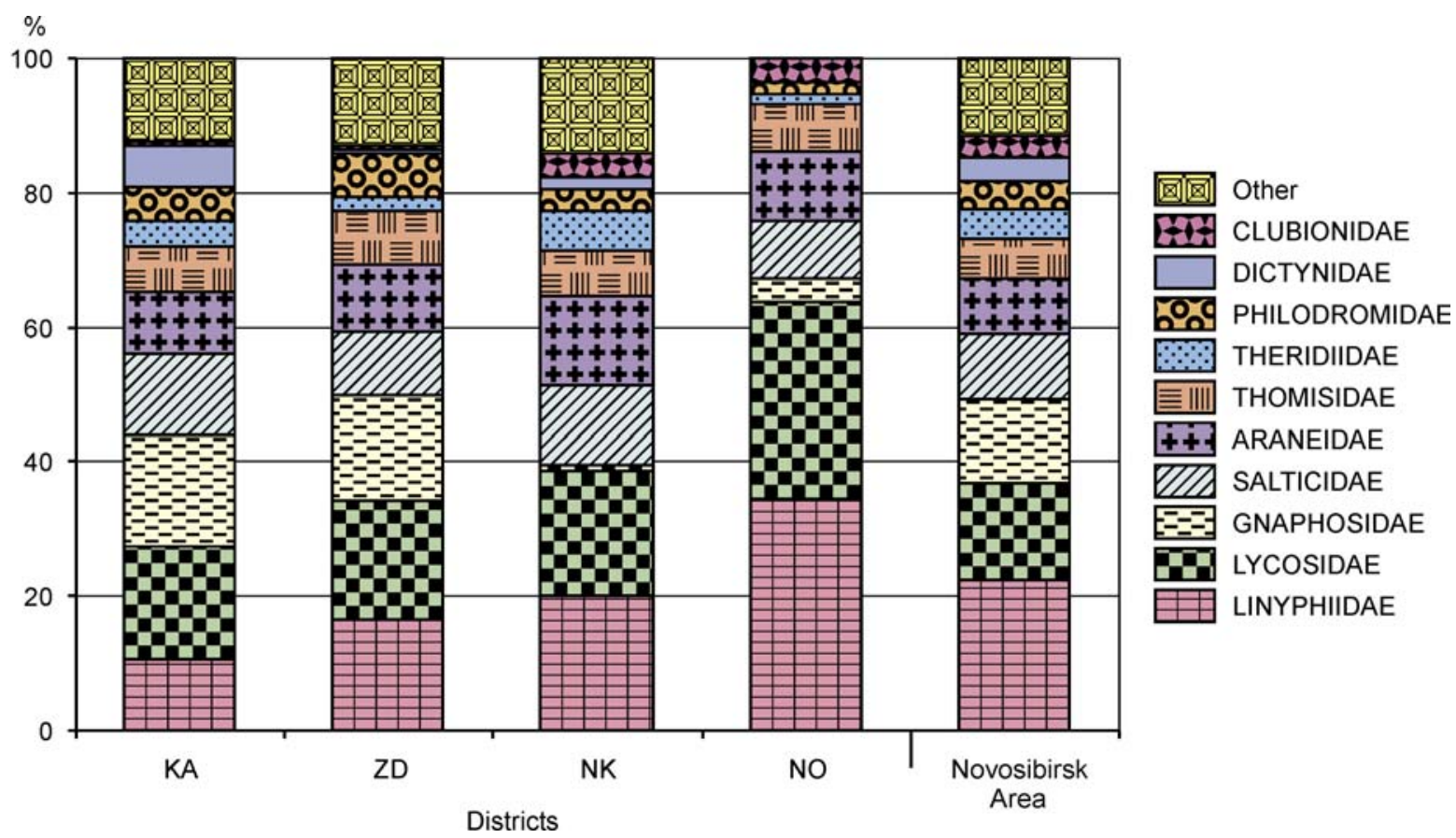

Fig. 5. Relative species diversity of spider families in Novosibirsk Area. Abbreviations: see Map.

Рис. 5. Соотношение видового богатства семейств пауков в Новосибирской области. Сокращения: см. Карту.

number of species having north- or north-easternmost limits of their distribution in Novosibirsk Area show the importance of the latitudinal (=zonal) zoogeographic barrier occurring in its territory; yet, it is also indicative of a similarity between the spider faunas of the southern part of Novosibirsk Area and Kazakhstan. It is also possible that the penetration of southern species northwards could be related to the impact of global warming and aridization of the climate, as was shown for other invertebrate groups [Bespalov et al., 2010]. New records of the spider species described from Tuvan steppe hollows having the westernmost limits of their distribution in Novosibirsk Area (e.g., Dictyna ubsunurica Marusik et Koponen, 1998 and Gnaphosa tuvinica Marusik et Logunov, 1992) are of great interest from the zoogeographic viewpoint, for West-Siberian steppes are much more humid than the sharpcontinental dry steppes of South Siberia; furthermore, at present they have no direct physical connections.

\section{Taxonomic diversity of spider families}

According to the present data, the family Linyphiidae is richest in Novosibirsk Area (82 species, 22,3\%), as in all regions beyond the latitude of $50^{\circ}$. Further arachnological studies may result in a markedly higher proportion of the linyphiids (30-40\%, or higher), but even the current number is evidence of a rather good state of knowledge of the regional spider fauna. Representatives of this family are renowned for their secluded and sedentary lifestyle, requiring special collecting methods.
The second most diverse family is Lycosidae (52 species, 14,3\%). Since the majority of contemporary collecting was carried out by means of pitfall traps, which is the most productive collecting method for the lycosids, this family seems to have been best inventoried. For the same reason, the Gnaphosidae are the third most diverse (=best studied) spider group in the Region (45 species, 12,3\%). As shown by long-term studies for many Siberian araneofaunas, the Lycosidae usually represent $7-10 \%$ of a total local diversity [Marusik, 2007]. Based on such proportion, it can be speculated that the total spider diversity of Novosibirsk Area is likely to be 500-750 species.

Ten spider families are represented by more than ten species. Besides the aforementioned families, seven others are as follows (in descending order of their regional diversity): Salticidae, Araneidae, Thomisidae, Theridiidae, Philodromidae, Dictynidae and Clubionidae (see Table 1). The latter order is virtually identical to that based on cumulative data on spiders of Asian Russia, Central Europe or the Nearctics [Marusik, 2007]. Proportions of spider families in the regional araneofauna are shown in Fig. 5.

Despite spider collecting has been undertaken in all 30 administrative units of Novosibirsk Area (see Map), its territory remains inventoried unevenly. Only 14 districts are known to have 16 or more spider species registered to date, and only in four of them more than 40 species: Karasuk Distr. - 178, Zdvinsk Distr. 140, City of Novosibirsk - 119, and Novosibirsk Distr. - 58. The araneofauna of Novosibirsk Distr. is least studied, which is also evident from a less number 
Table 2. Representation of spider species with various range types in the araneofauna of Novosibirsk Area. Abbreviations as in Table 1 .

Таблица 2. Представленность видов пауков с разными типами ареалов в аранеофауне Новосибирской области. Обозначения как в таблице 1 .

\begin{tabular}{|l|c|c|c|c|c|c|}
\hline & TH & TP & WP & CP & EP & Total \\
\hline B & 7 & 16 & 6 & 5 & 2 & 36 \\
\hline SH & 19 & 57 & 78 & 5 & 5 & 164 \\
\hline SA & 0 & 0 & 22 & 9 & 1 & 32 \\
\hline P & 44 & 52 & 30 & 0 & 3 & 129 \\
\hline Total & 72 & 125 & 134 & 19 & 11 & 361 \\
\hline
\end{tabular}

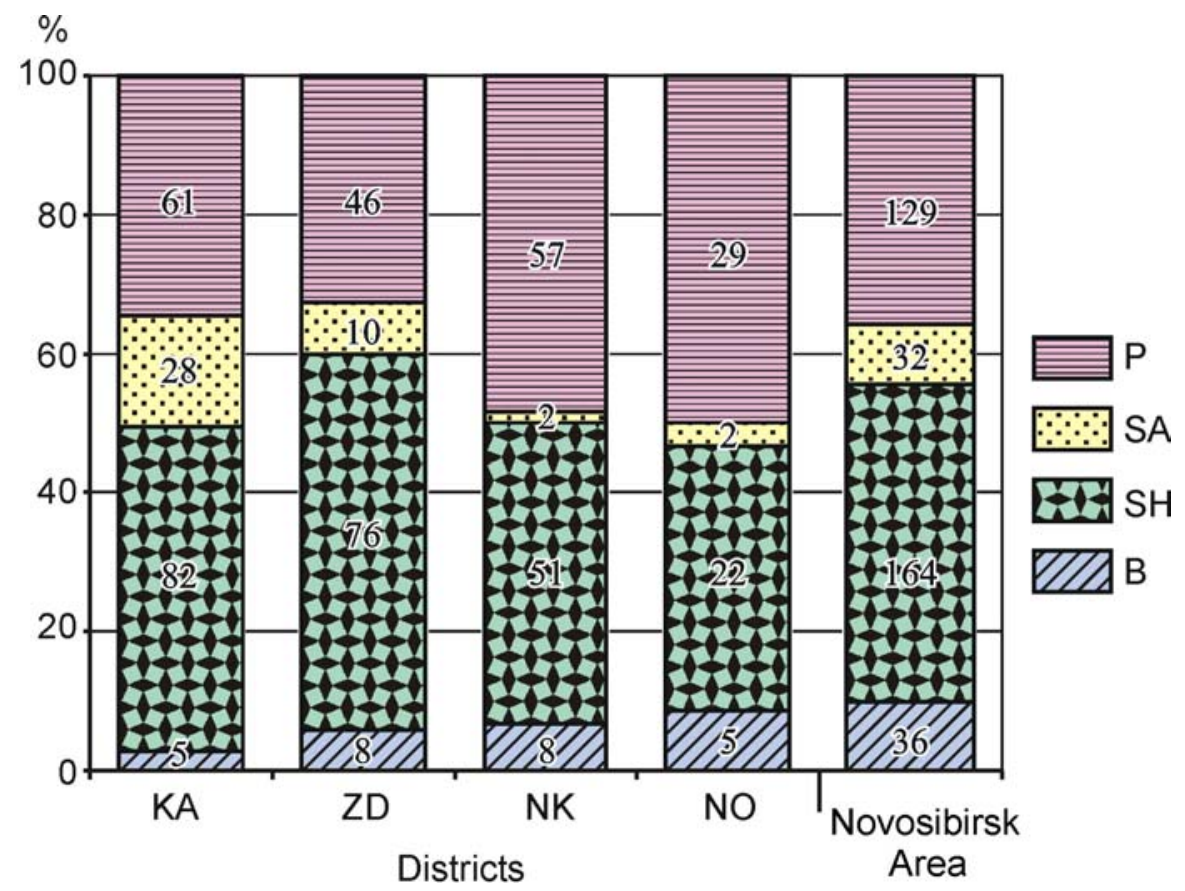

Fig. 6. Proportions of spider species with various latitudinal components in large faunas of Novosibirsk Area. Abbreviations: see Map.

Рис. 6. Доля видов пауков с разной широтной составляющей ареала в крупных фаунах Новосибирской области. Сокращения: см. Карту.

of the registered spider families. In the arid Karasuk Distr. the higher proportion of Salticidae, Gnaphosidae and Dictynidae has been registered, and the markedly smaller one of Linyphiidae. Contrary to that, the araneofaunas of City of Novosibirsk and Novosibirsk Distr. are deprived of the gnaphosids (1-2 species), with over $60 \%$ being represented by the linyphiids and lycosids. In City of Novosibirsk, a relatively high proportion of families with 1-2 species, including synathropic species, has been recorded (Fig. 5).

\section{Zoogeographic analysis of local faunas}

Latitudinally, the majority of spiders recorded from Novosibirsk Area belong to the subboreal humid (45\%) or polyzonal (36\%) groups (see Table 2 ), which can be explained by the predominance of forest-steppes on the territory at hand. These latitudinal groups basically consist of west-Palaearctic and trans-Palaearctic widespread species. A significant share is also made up by boreal and subarid species (9-10\%) of which the majority have been collected from intrazonal landscapes of the Ob' River basin and Salair Ridge; boreal species were also recorded from the northern part of the studied region, while subarid ones from the steppe zone (see Map). Over a half of the boreal species belongs to the trans-Palaearctic and trans-Holarctic longitidinal groups, whereas all the subarid species are represented by west- and central-Palaearctic species.

Longitudinaly, west-Palaearctic spider species predominate (37\%), probably because West Siberian Plain lies in the eastern periphery of the West Palaearctics. 


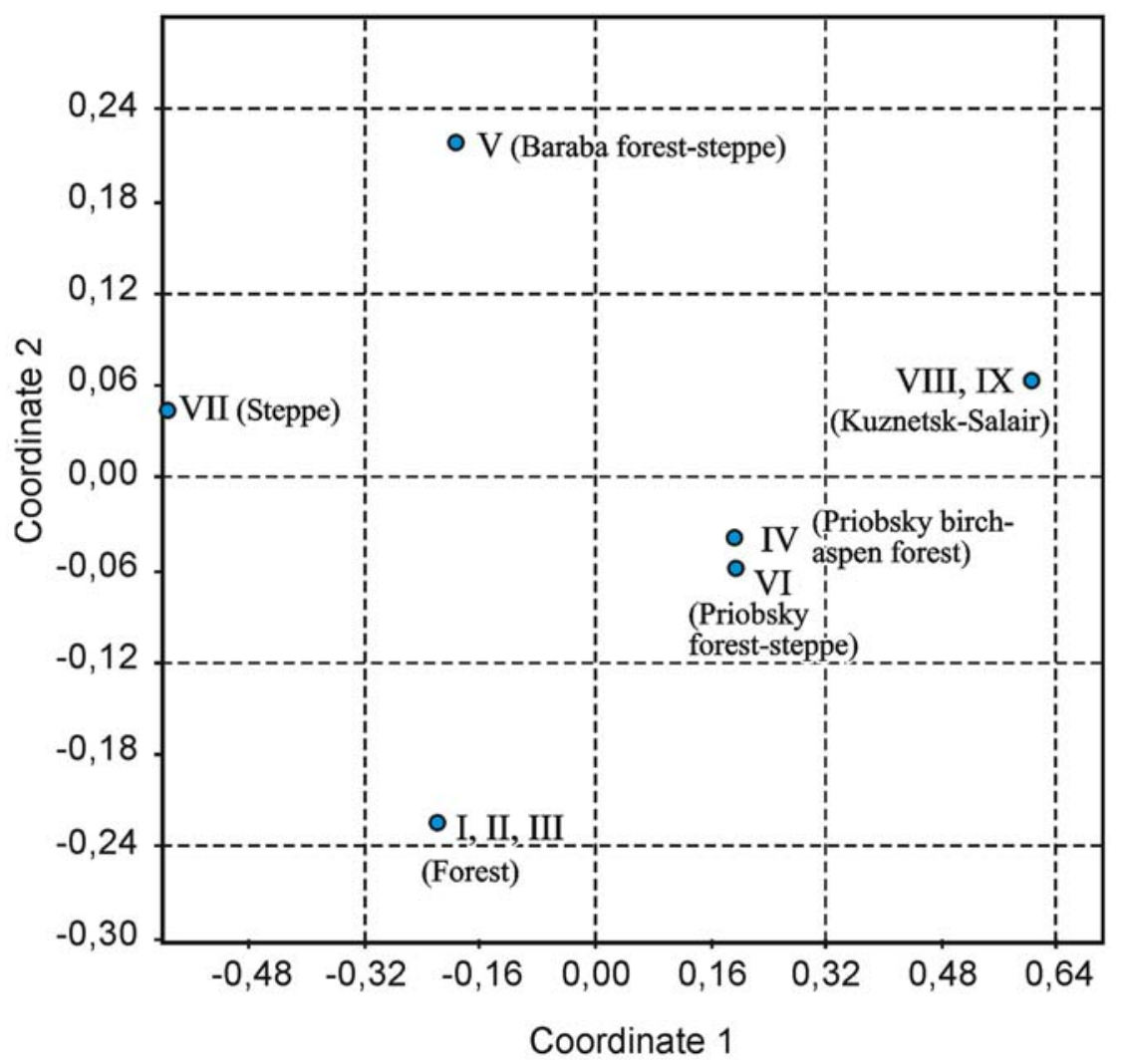

Fig. 7. Similarity of some landscape zones of Novosibirsk Area based on the spider fauna. Szymkiewicz -Simpson's similarity index, multidimensional scaling (MDS).

Рис. 7. Сходство некоторых ландшафтных районов Новосибирской области по фауне пауков. Коэффициент сходства Шимкевича-Симпсона, многомерное шкалирование (MDS).

Yet, a significant share is made up by trans-Palaearctic $(34 \%)$ and trans-Holarctic (19\%) species. The share of east-Palaearctic species is low $(5 \%)$, suggesting that the westward penetration of eastern species has taken place to a much smaller degree than the eastward one (western species to the east).

If the range compositon of ground beetles [see Dudko \& Lyubechanskii, 2002: Table 3] and spiders are compared, the main difference seems to be a much larger share of polyzonal species in spiders $(36 \% \mathrm{vs}$. $12 \%$ in the carabids), which is achieved by reducing the number of boreal and subarid species. If longitudinal components are considered, spiders differ from the Carabidae in having much larger shares of trans-Holarctic $(19 \%$ vs. 5,6\%) and trans-Palaearctic (34\% vs. $20 \%$ ) species. The share of east-Palaearctic species is about the same in both groups $(5 \%$ vs. $8 \%)$. Such differences in the range compositon of Aranei and Carabidae are likely to be explained by the history of their fauna formations during the last few thousands of years [Clarke et al., 2001].

As seen in Fig. 6, of the four better studied local araneofaunas, those of south-western districts of Kara- suk and Zdvinsk are characterized by the larger share of subarid species. Yet, the share of such species is markedly higher in Karasuk than in Zdvinsk. This is expected, for Karasuk Distr. lies to the south of Zdvinsk (Map), already in the steppe zone rather than in the forest-steppe. In the meadow Zdvinsk Distr., the highest proportion goes to subboreal humid species, even exceeding those in the faunas of Novisibirsk Distr. and City of Novosibirsk. In two last districts polyzonal species predominate, with the share of boreal species being also visible. The entire araneofauna of Novosibirsk Area is characterized by almost equal shares of subarid and boreal species, as well as those of subboreal humid and polyzonal species, thus somewhat resembling the graph of Zdvinsk Distr.

Based on an analysis of longitudinal components, west-Palaearcrtic species predominate in the entire arachnofauna of Novosibirsk Area, accounting for almost a third of the species diversity (120 species in total). Such observation is consistent with the trend revealed for other invertebrate groups of Novosibirsk Area, for instance, the ground beetles of which about a 
half display a west-Palaearcrtic distributional pattern [Dudko, Lyubechanskii, 2002].

\section{Araneo-complexes of Novosibirsk Area}

Not all the landscape subzones have been inventoried equally from the araneological viewpoint. Only four of them contain 156 or more registered spider species: viz., Priobsky birch-aspen forest (IV), Baraba forest-steppe (V), Priobsky forest-steppe (VI), and Steppe Province (VII) (see Map). The Baraba foreststeppe and Steppe Provinces have the comparatively well-studied araneofaunas (Zdvinsk and Karasuk districts correspondingly), whereas others show a large number of common species. Having united the Vasyugan taiga-swampy (I), Priobsky south taiga (II) and North Baraba sub-taiga (III) subzones, we have resulted in the 'integrated forest subzone' from which only 55 spider species have been registered to date. The Kuznetsk-Salair Province (VIII and XI) represents a small sector of the studied territory, with its araneofauna remaining practically unknown to date (only 32 species).

Despite the incompletness of our knowledge, the accepted physiogeographic subdivision of Novosibirsk Area (see Map) seems to be adequate for analyzing the studied spider fauna. Based on the MDS ordination (Fig. 7), the six landscape subzones are grouped in two clusters: one consists of the territories being situated on the left bank of Ob' River, whereas the other includes subzones lying on its right bank. Yet, the araneofaunas of Baraba forest-steppe (V) and the 'integrated forest subzone' (I, II and III) are similar by the first axis, while are clearly distinct by the second axis. In the right bank cluster, an anticipated similarity of Priobsky faunas is observed, except for the standing apart araneofauna of the Salair Ridge.

The zoogeographic regioning of Novosbirsk Area based on local faunas of the ground-beetles (Carabidae) [Dudko, Lyubechanskii, 2002] looks very similar to that based on spiders. Their fauna is clearly divided along Ob' River into the eastern and western clusters; the steppe province is also distinct in the western cluster. As with spiders, the fauna of groundbeetles of $\mathrm{Ob}$ ' region is monolithic. Thus, the factors affecting biogeographical distribution of these two taxonomically unrelated groups of terrestrial predatory arthropods are likely to be very similar.

ACKNOWLEDGEMENTS. We are grateful to O.E. Kosterin, N.V. Priydak, A.A. Fomichev (all from Novosibirsk, Russia) for giving access to the newly collected spider materials; S.L. Esyunin (Perm, Russia), D.V. Logunov (Manchester, UK) and A.V. Gromov (Bingen am Rhein, Germany) for providing their unpublished data; S.L. Esyunin, D.V. Logunov and K.G. Mikhailov (Moscow, Russia) for valued comments during the preparation of this paper and enormous help (KGM) with literature; V.V. Ivonin (No- vosibirsk, Russia) for help with old literature; A.V. Tanasevitch (Moscow, Russia) for the identification of some Linyphiidae; T.P. Ryabikova (Novosibirsk, Russia) for the help with explaining some old localities. We also wish to thank S.L. Esyunin and Yu. M. Marusik who kindly read the first draft and provided the critical comments, helping us to improve the ms. Finally, we are thankful to D.V. Logunov for his linguistic help.

The study was supported by the Russian Foundation for Basic Research (grant No. 15-04-07591a) and the Federal Agency for Scientific Organizations programme for support the bioresource collections (No. AAAA-A17-117101070002-6).

\section{References}

Atlas of Novosibirsk Region (2nd Edition). 2002. Moscow-Novosibirsk: Roskartografiya. 56 pp. [In Russian]

Azarkina G.N., Logunov D.V. 2001. Separation and distribution of Xysticus cristatus (Clerck, 1758) and X. audax (Schrank, 1803) in eastern Eurasia, with description of a new species from the mountains of central Asia (Aranei: Thomisidae) // Arthropoda Selecta. Vol.9. No.2. P.133-150.

Azarkina G.N., Marusik Y.M., Antonenko T.V. 2015. First description of the male of Alopecosa azsheganovae Esyunin, 1996 (Araneae: Lycosidae) // Zootaxa. Vol.4033. No.2. P.265269. Doi: 10.11646/zootaxa.4033.2.5

Azarkina G.N., Trilikauskas L.A. 2012. Spider fauna (Aranei) of the Russian Altai, part I: families Agelenidae, Araneidae, Clubionidae, Corinnidae, Dictynidae and Eresidae // Euroasian Entomological Journal. Vol.11. No.3. P.199-208+212+I.

Azarkina G.N., Trilikauskas L.A. 2013a. Spider fauna (Aranei) of the Russian Altai, part II: families Gnaphosidae, Hahniidae, Linyphiidae, Liocranidae and Lycosidae // Euroasian Entomological Journal. Vol.12. No.1. P.51-67.

Azarkina G.N., Trilikauskas L.A. 2013b. Spider fauna (Aranei) of the Russian Altai, part III: families Mimetidae, Miturgidae, Oxyopidae, Philodromidae, Pholcidae, Pisauridae, Salticidae, Sparassidae, Tetragnathidae, Theridiidae, Thomisidae, Titanoecidae, Uloboridae and Zoridae // Euroasian Entomological Journal. Vol.12. No.3. P.243-254.

Bespalov A.N., Dudko R.Y., Lyubechanskii I.I. 2010. [Additions to the ground beetle fauna (Coleoptera, Carabidae) of the Novosibirsk Oblast: do the southern species spread to the north?] // Euroasian Entomological Journal. Vol.9. No.4. P.625-628 [in Russian, with English summary].

Chugunov S., Chugunov M. 1902. [Entomological observations during a zoological expedition between the $\mathrm{Ob}$ and Irtysh Rivers in summer 1899] // Estestvoznanie i geografiya. Vol.7. No.2. P.32-40 [in Russian].

Clarke T.E., Levin D.B., Kavanaugh D.H., Reimchen T.E. 2001. Rapid evolution in the Nebria gregaria group (Coleoptera: Carabidae) and the paleogeography of the Queen Charlotte Islands // Evolution. Vol.55. No.7. P.1408-1418.

Danilov S.N., Logunov D.V. 1994. Faunistic review of the jumping spiders of Transbaikalia (Aranei: Salticidae) // Arthropoda Selecta. Vol.2. No.4. P.25-39.

Dudko R.Yu., Lyubechanskii I.I. [Faunal and zoogeographic analysis of the ground beetles (Coleoptera, Carabidae) of Novosibirsk Oblast'] // Euroasian Entomological Journal. 2002. Vol.1. No.1. P.30-45 [in Russian, with English summary].

Emelyanov A.F. 1974. [Proposals for the classification and nomenclature of ranges] // Entomologicheskoe obozrenie. Vol.53. No.3. P.497-522 [in Russian].

Ermolaev V.N. 1928. Materialen zur Spinnenfauna Westsibiriens I-II // Archiv für Naturgeschichte. Vol.92A (1926).No.7. P.97111.

Ermolaev V.N. 1930. [To the problem of Siberian distribution of a tarantula, Hogna (Trochosa) singoriensis (Laxm.)] // Russkoe 
entomologicheskoe obozrenie. Vol.24. No.3-4. P.212-217 [in Russian].

Eskov K.Y., Marusik Y.M. 1992. The spider genus Centromerus (Aranei, Linyphiidae) of the fauna of siberia and the Russian Far East, with analysis of its distribution // Arthropoda Selecta. Vol.1. No.2. P.33-46.

Eskov K.Y., Marusik Y.M. 1994. New data of the taxonomy and faunistics of North Asian linyphiid spiders (Aranei, Linyphiidae) // Arthropoda Selecta. Vol.2. No.2. P.41-79.

Fyodorov I. V., Trilikauskas L. A. 2013. [Spider population of a neogenic ecosystem of the salt lake arid zone in Kulunda, West Siberia, Russia] // Eurasian Entomological Journal. Vol.12. No.3. P.233-242 [in Russian].

Hammer Ø., Harper D.A.T., Ryan P.D. 2001. PAST: Paleontological statistics software package for education and data analysis / / Palaeontologia Electronica. Vol.4. No.1. P.9.

Huber B. 2011. Revision and cladistic analysis of Pholcus and closely related taxa (Araneae, Pholcidae) // Bonner Zoologische Monographien .Vol.58. P.1-509.

Lavrova N.M. 1923. [Fauna of the Karachi Lake] // Izvestiya Tomskogo universiteta. Vol.72. P.1-8 [in Russian, with German summary].

Litvinchuk L.N. 1980. [The sawfly, Pamphilus vafer]. Novosibirsk: Nauka. 48 pp. [In Russian]

Levi H.W. 1967. Cosmopolitan and pantropical species of theridiid spiders (Araneae: Theridiidae) // Pacific Insects. Vol.9. P.175186.

Lobanova T.V. 1976. [On a species composition of the wolf spiders (Aranei, Lycosidae) in West Siberia] // Fauna gel'mintov chlenistonogikh Sibiri. Trudy Biologicheskogo instituta Sibirskogo otdeleniya AN SSSR. Vol.18. P.47-66 [in Russian].

Lobanova T.V. 1980. [On the spider (Aranei, Lycosidae) population of the berry-fruit orchards of Novosibirsk Region] // Zhivotnyi mir Sibiri i ego okhrana. Novosibirsk: Novosibirski pedagogicheskii institut. P.16-18 [in Russian].

Lobanova T.V., Ryabikova T.P. 1980. [On parasites and predators of the spider family Lycosidae] // Zhivotnyi mir Sibiri i ego okhrana. Novosibirsk: Novosibirskii pedagogicheskii institut. P.21-24 [in Russian].

Logunov D.V. 1990. [New data on spiders of the families Atypidae, Pisauridae and Thomisidae of the USSR fauna] // Chlenistonogie i gel'minty. Novosibirsk: BI SA RAN. P.33-43 [in Russian].

Logunov D.V. 1992a. The spider family Salticidae (Araneae) from Tuva. II. An annotated check list of species // Arthropoda Selecta. Vol.1. No.2. P.47-71.

Logunov D.V. 1992b. [A review of the spider genus Tmarus Simon, 1875 (Araneae, Thomisidae) in the USSR fauna, with a description of a new species] // Sibirskii biologicheskii zhurnal. Vol.1. P.61-73 [in Russian, with English summary].

Logunov D.V. 1996. A critical review of the spider genera Apollophanes O. Pickard-Cambridge, 1898 and Thanatus C.L. Koch, 1837 in North Asia (Araneae, Philodromidae) // Revue Arachnologique. T.11. Fasc.13. P.133-202.

Logunov D.V. 1999. Redefinition of the genera Marpissa C. L. Koch, 1846 and Mendoza Peckham \& Peckham, 1894 in the scope of the Holarctic fauna (Araneae, Salticidae) // Revue Arachnologique. Vol.13. P.25-60.

Logunov D.V., Cutler B., Marusik Yu.M. 1993. A review of the genus Euophrys C. L. Koch in Siberia and the Russian Far East (Araneae: Salticidae) // Annales Zoologici Fennici. Vol.30. P.101-124.

Logunov D.V., Heçiak S. 1996. Asianellus, a new genus of the subfamily Aelurillinae (Araneae: Salticidae) // Entomologica Scandinavica. Vol.26. P.103-117.

Logunov D.V., Kronestedt T. 1997. A new Palearctic species of the genus Sitticus Simon, with notes on related species in the floricola group (Araneae, Salticidae) // Bulletin of the British Arachnological Society. Vol.10. P.225-233.

Logunov D.V., Marusik Yu.M. 1994. New data on the jumping spiders of the Palearctic fauna (Aranei Salticidae) // Arthropoda Selecta. Vol.3. No.1-2. P.101-115.

Logunov D.V., Marusik Yu.M. 1998. A new species of the genus Xysticus from the mountains of south Siberia and Mongolia
(Araneae, Thomisidae) // Bulletin of the British Arachnological Society. Vol.11. Pt.3. P.103-106.

Logunov D.V., Marusik Yu.M. 1999 (for 1998). A brief review of the genus Chalcoscirtus Bertkau, 1880 in the faunas of Central Asia and the Caucasus (Aranei: Salticidae) // Arthropoda Selecta. Vol.7. No.3. P.205-226.

Logunov D.V., Marusik Yu.M. 2000a (for 1999). Miscellaneous notes on Palaearctic Salticidae (Arachnida: Aranei) // Arthropoda Selecta. Vol.8. No.4. P.263-292.

Logunov D.V., Marusik Yu.M. 2000b. Catalogue of the jumping spiders of northern Asia (Arachnida, Araneae, Salticidae). Moscow: KMK Scientific Press Ltd. 299 pp.

Logunov D.V., Rakov S.Yu. 1996. A review of the spider genus Synageles Simon, 1876 (Araneae, Salticidae) in the fauna of Central Asia // Bulletin de l'Institut Royal des Sciences Naturelles de Belgique, Entomologie. Vol.66. P.65-74.

Logunov D.V., Weso ${ }^{3}$ owska W. 1995. New data on some poorly known Palaearctic species of Sitticus (Araneae: Salticidae) // Genus. Vol.6. P.163-175.

Lyubechanskii I.I., Azarkina G.N. 2017. Ecological structure of the west Siberian forest-steppe spider community (Arachnida, Araneae) and its comparison with the ground-beetle (Coleoptera, Carabidae) community // Contemporary Problems of Ecology. Vol.10. No.2. P.164-177; doi: 10.1134/S1995425517020081

Lyubechanskii I.I., Azarkina G.N., Dudko R.Yu. 2017. [Soil-dwelling predatory arthropod community (Insecta: Carabidae; Arachnida: Araneae; Opiliones) in the valley ecosystem of the Upper $\mathrm{Ob}$ ' River at the beginning and the end of seasonal activity period] // Euroasian Entomological Journal. Vol.16. No.2. P.141-150 [in Russian, with English summary].

Marusik Yu.M. 2007. Spiders (Arachnida, Aranei) of the Asian Part of Russia. Autoreferat of Dr. Sci. Thesis. Saint-Petersburg. Saint-Petersburg State University. 40 pp. [In Russian]

Marusik Yu.M., Gnelitsa V.A., Koponen S. 2007. A survey of Holarctic Linyphiidae (Aranei). 4. A review of the erigonine genus Lophomma Menge, 1868 // Arthropoda selecta. Vol.15. No.2. P. $153-171$.

Marusik Yu.M., Logunov D.V. 1998. Taxonomic notes on the Evarcha falcata species complex (Aranei Salticidae) // Arthropoda Selecta. Vol.6. No.3-4. P.95-104.

Marusik Yu.N., Logunov D.V., Koponen S. 2000. Spiders of Tuva, South Siberia. Magadan: IBPN FEB RAS. 252 pp.

Marusik Yu.M., Omelko M.M. 2014. A survey of East Palaearctic Gnaphosidae (Araneae). 3. On new and poorly known Gnaphosa Latreille, 1804 // Zootaxa. Vol.3894. No.1. P.10-32.

Mikhailov K.G. 1992. The spider genus Clubiona Latreille, 1804 (Arachnida Aranei Clubionidae) in the USSR fauna: a critical review with taxonomical remarks // Arthropoda Selecta. Vol.1. No.3. P.3-34.

Mikhailov K.G. 1996. A checklist of the spiders of Russia and other territories of the former USSR // Arthropoda Selecta. Vol.5. No.1-2. P.75-137.

Mikhailov K.G. 2003. The spider genus Clubiona Latreille, 1804 (Aranei: Clubionidae) in the fauna of the former USSR: 2003 update // Arthropoda Selecta. Vol.11. P.283-317.

Mikhailov K.G. 2013. The spiders (Arachnida: Aranei) of Russia and adjacent countries: a non-annotated checklist // Arthropoda Selecta. Supplement No.3. Moscow: KMK Scientific Press Ltd. 262 p.

Mordkovich V.G., Dudko R.Yu., Trilikauskas L.A., Lyubechanskii I.I. 2015. [Carabid beetles (Coleoptera, Carabidae) and spiders (Aranei) are part of the soil fauna on the shore of a salt like in South Siberia, Russia] // Euroasian Entomological Journal. Vol.14. No.5. P.447-454 [in Russian, with English summary].

Ovtsharenko V.I., Platnick N.I., Song D.X. 1992. A review of the North Asian ground spiders of the genus Gnaphosa (Araneae, Gnaphosidae) // Bulletin of the American Museum of Natural History. Vol.212. P.1-88.

Rakov S.Yu. 1999. On the spider fauna of jumping spiders of the southern part of West Siberia // Arthropoda Selecta. Vol.6. No.1-2. P.105-112.

Ruzski M.D. 1924. [Materials on the biology of the Karachi Lake and Health Resort (a preliminary communication)] // Kurort- 
noe delo. Vol.2. No.7-8. P.3-6 [in Russian, with German summary].

Ruzski M.D. 1925. [Materials on the fauna of the "Karachi Lake" Health Resort] // Izvestiya Tomskogo universiteta. Vol.75. P.283-290 [in Russian].

Ruzski M.D. 1928. [On the fauna of the "Karachi" Health Resort and its environs] // Sbornik bal'neologicheskikh rabot po Sibirskim kurortam. Tomsk. Vol.2. P.101-106 [in Russian].

Ruzski M.D. 1946. [Zoodynamics of the Baraba Steppe] // Trudy Tomskogo universiteta. Vol.97. Seriya biologicheskaya. Voprosy zoologii. P.17-68 [in Russian].

Ryabikova T.P. 1985. [To the fauna of the orb-weaving spider (Aranei, Araneidae) in the South of West Siberia] // Pauki i nasekomye Sibiri. Novosibirsk: NGPI. P.9-13 [in Russian].

Ryabikova T.P. 1989. [Species composition and biology of the orbweaving spiders (Aranei, Araneidae) in the south of West Siberia] // Fauna, ekologiya and zoogeografiya pozvonochnykh i chlenistonogikh. Novosibirsk: NGPU. P.135-149 [in Russian].

Ryabikova T.P., Lobanova T.V. 1980. [Materials to the population of the spider families Araneidae and Linyphiidae in the environs of Novosibirsk Town] // Zhivotnyi mir Sibiri i ego okhra- na. Novosibirsk: Novosibirskii pedagogicheskii institute. P.1921 [in Russian].

Szita É., Logunov D.V. 2008. A review of the histrio group of the spider genus Philodromus Walckenaer, 1826 (Araneae, Philodromidae) of the eastern Palaearctic region // Acta Zoologica Academiae Scientiarum Hungaricae. Vol.54. P.23-73.

Tanasevitch A.V. 2000. On some Palaearctic species of the spider genus Agyneta Hull, 1911, with description of four new species (Aranei: Linyphiidae) // Arthropoda Selecta. Vol.8. No.3. P.201-213.

Trilikauskas L.A., Dudko R.Yu. 2016. [On the late spring aspect of the spider (Arachnida, Aranei) and ground beetle (Coleoptera, Carabidae) populations in coniferous forests of the south-east part of the West Siberian Plain (Novosibirsk Region)] // Vestnik Tomskogo gosudarstvennogo universiteta. Biologiya - Tomsk State University Journal of Biology. Vol.2. No.34. P.114 125 [in Russian, English summary]; doi: 10.17223/19988591/ $34 / 8$

World Spider Catalog. 2017. World Spider Catalog. Natural History Museum Bern, online at http://wsc.nmbe.ch, version 18.5 (accessed on 19 December 2017); doi: 10.24436/2.

Responsible editor D.V. Logunov 